\title{
Analysing the Vulnerability of Public Transport Networks
}

\author{
Mary Luz Mouronte-López $\mathbb{D}$ \\ Higher Polytechnic School of Universidad Francisco de Vitoria, Pozuelo de Alarcón, Madrid 28223, Spain \\ Correspondence should be addressed to Mary Luz Mouronte-López; maryluz.mouronte@ufv.es
}

Received 13 January 2021; Revised 17 February 2021; Accepted 25 February 2021; Published 11 March 2021

Academic Editor: Sebastian Wandelt

Copyright ( $) 2021$ Mary Luz Mouronte-López. This is an open access article distributed under the Creative Commons Attribution License, which permits unrestricted use, distribution, and reproduction in any medium, provided the original work is properly cited.

\begin{abstract}
This paper analyses the robustness of specific public transport networks. Common attributes and which of them have more influence on the networks' vulnerability are established. Initially, the structural properties of the networks in two graphical representations (L-Space and P-Space) are checked. Afterwards, the spread of problems (traffic jams, etc.) are simulated, employing a model based on a propagation and recovery mechanism, similar to those used in the epidemiological processes. Next, the size of the largest connected subset of stops of the network (giant component) is measured. What is shown is that the faults randomly happened at stops or links, also displaying that those that occurred in the highest weighted links spread slower than others. These others appear at stops with the largest level of betweenness, degree, or eigenvector centralities and PageRank. The modification of the giant component, when several stops and links are removed, proves that the removal of stops with the highest interactive betweenness, PageRank, and degree centralities has the most significant influence on the network's integrity. Some equivalences in the degree, betweenness, PageRank, and eigenvector centrality parameters have been found. All networks show high modularity with values of index $Q$ close to 1 . The networks with the highest assortativity and lowest average number of stops are the ones which a passenger can use to travel directly to their destination, without any change. The Molloy-Reed parameter is higher than 2 in all networks, demonstrating that high integrity exists in them. All stops were characterized by low $k$-cores $\leq 3$.
\end{abstract}

\section{Introduction}

The question of vulnerability of a public transport network (PTN) against problems in nodes or links is a matter of high interest. All nodes and links may accidentally fail with a similar probability, but the severity of the damage caused is higher when it occurs in some nodes or links rather than in others. The PTN is a key issue in the main cities of the world, having to operate efficiently on a constant basis. They must display a high tolerance to problems, keep the possible delays to a minimum, and manage the duration of a journey in accordance with the trip planning. With the purpose of persuading passengers to make use of the PTN, some strategies should be implemented to ensure the continued running of networks, the prevention of problems before, and the mitigation of losses during and after the failure $[1,2]$.

This paper analyses the vulnerability of some PTNs. The study firstly focuses on the analysis of the structural properties of the PTN (betweenness centrality, degree, eigenvector centrality, PageRank, average path length, $k$ core, modularity, and assortativity). Next, the fault propagation is simulated (e.g., traffic jams caused by accidents or roadworks), employing different selection criteria of nodes and links, in order to detect the stops in whose immediate vicinity congestion is more prone to happening. A procedure based on epidemic spread algorithms is used. The effect of the structure of the network on the failure propagation is also examined. Finally, the size of the largest connected subset of stops of the PTN (giant component, GC) is estimated, which is calculated when several stops and links are disconnected according to certain criteria. Depending on the value of this magnitude, a serious issue could occur due to the existence of inaccessible routes (construction work or redesign activities in networks), which cause unavoidable stops or important delays during a trip. The analysis allows the identification of the most critical stops and links of a PTN and establishes a subnetwork of highly connected stops. 
Ten networks are analysed. They are Auckland in New Zealand, which consists of 5223 stops and 318,896 links; EMT in Madrid, Spain, which has 4636 stops and 2,070,508 links; BC Transit in Vancouver, Canada, which consists of 3981 stops and 702,197 links; Kolumbus in Rogaland, Norway, which has 3828 stops and 487,432 links; AVL, CFL, RGTR, and TICE in Luxembourg which contains 1372 nodes and 340,684 links; STAR in Rennes, France, which consists of 1415 stops and 9,477,213 links; Thunder Bay Transit, in Ontario, Canada, which contains 825 nodes and 78,247 links; TransAntofagasta, in Chile, which has 650 nodes and 724,362 links; Linja-Karjala Oy, in Kuopio, Finland, which contains 551 nodes and 63,339 links; and finally, CIT Chambly-Richelieu-Carignan, in Quebec, Canada, which consists of 346 nodes and 9366 links. The reason for the selection was to have networks of various sizes (small, medium, and large) and topologies.

Specifically, the goals of this research are as follows:

(1) Analysis of the robustness of some PTNs: detection of commonalities and key aspects.

(2) Discovery of properties on a network's vulnerability that can be generalized.

Several investigations exist about PTN, some of them describing a comprehensive survey of statistical properties of PTN based on the data of several cities, and at the same time, presenting models that reproduce the best part of their properties [3]. Other research detects the critical nodes in PTN in order to enable contingency mechanics in them.

Berche et al. [4] studied the PTN's resilience applying different attack scenarios, which range from random failure to targeted destruction. Some nodes are removed according to certain operating characteristics. The candidate nodes to be eliminated were selected according to their highest degree, closeness, graph, stress, and betweenness centralities, as well as the largest clustering coefficient and next nearest neighbouring number. In [5], an explanation exists of a procedure for the detection of critical airports in the global air transport network (ATN), which is based on simulating an attack on specific airports using several adaptive selection criteria. Wandelt et al. [6] evaluated several strategies for dismantling of networks and identified large heterogeneities in their performance. The authors show that the use of the interactive betweenness creates far stronger attacks. Because of the high complexity of this algorithm, the authors recommend its use on small networks. After, in [7], the authors propose approximations to interactive betweenness in order to improve the computational cost of the algorithm. Other research analyses the effect that the addition of new links has on the robustness of the networks or design methodologies in order to detect the most vulnerable links in PTN [8].

The robustness of a network can be estimated in terms of passenger welfare [9]. Additionally, the accessibility of the PTN is evaluated by several types/examples of research. Albacete et al. [10] compared and applied two location-based methods to analyse PTN accessibility. The results had considerable implications for transport policy making. In [11], a thorough assessment is carried out, focusing on the possible applications of an optimization-based scheme for the building of several timetables and proactive railway traffic management. This is carried out over a large network, employing stochastic disturbances. Wandelt et al. [12] proposed a framework to evaluate and improve the robustness of transportation systems by exploiting the existence of communities. The method is applied to several realworld transportation systems. Sun et al. [13] studied the resilience of cities when disruptions in their airports happen.

Relationships between robustness and accessibility are also studied $[14,15]$. Other authors suggested methods and spread models which analyse the performance of networks. He et al. [16] defined a mathematical model to evaluate the effect of the propagation of the risk of failure in multimodal transport networks. Baspinar and Koyuncu [17] proposed a new model for the air transportation network under stress and defined parameters in order to describe not only air sectors, but also airports, as well as flights. They used epidemic spreading processes by assuming that the characteristics of disease spreading and the delay propagation are similar. Akdere et al. [18] studied the reliable data dissemination in the context of wireless sensor network environments. It shows the applicability of epidemic spread algorithms in those environments and carries out a comparative performance analysis of several mechanisms in terms of message delivery rate, average message latency, and messaging overhead on the network. As other research suggests, Nekovee [19] presented a new epidemic algorithm for information dissemination in highly dynamic and intermittently connected vehicular ad hoc networks (VANET). It shows through realistic simulations of highway traffic that the proposed algorithm is suitable to achieve a reliable and efficient level of information transmission in a context with frequent network fragmentation and large density variations.

This paper is organized as follows: firstly, resources and methods used in this work are explained in detail. It focuses on the used data and the developed software programs, which correspond with the design of the network and the employed parameters to characterize the networks' topology. The propagation model and the study of evolution of the GC when certain elements are removed are also studied. Afterwards, the results, conclusions, and future projects are described and discussed.

\section{Materials and Methods}

2.1. Overview of Used Resources. Information of stops and routes of Auckland, EMT, BC Transit, Kolumbus, STAR, AVL, CFL, RGTR, TICE, Thunder Bay Transit, TransAntofagasta, Linja-Karjala Oy, and CIT networks was retrieved from public sources (see the Data Availability section for details). Several programs in R [20] and Python [21] were implemented, using the R.3.6.0 and 3.8.3 version, respectively. We use the Python networkx and pathpy packages and the igraph $\mathrm{R}$ package. The programs followed the typical development life cycle with stages of specification, design, and testing. 
2.2. Overview of Used Methods. The structural properties of the networks are analysed. A new propagation algorithm, which was designed and undertaken in the networks in order to simulate the spread of a failure, is detailed. This is followed by a description of how the evolution of the GC is calculated, with the aim of studying the specific impact a problem might have on each network.

2.3. Study of Structural Properties. A PTN can be represented in two topological spaces L-Space $[22,23]$ and P-Space $[2,24]$. In both spaces, the network is mapped as a graph $G=(N ; L)$, where $N$ is the set of nodes symbolizing the stops and $L$ is the set of links established between them. In L-Space, one node symbolizes one stop, and one link means a union between two consecutive stops, which tells us there is a link between two stops, if one stop is the successor of the other on a route. This space aims to show the geographical proximity between stops. In the P-Space [2, 24], one node represents one stop, and one link joins a pair of stops, if at least one route provides direct service between them. A link means that passengers can take at least one route for a direct trip between two stops. If travellers have to exchange routes, then the pair of stops is joined by more than one link. This space aims to show the transfer relationship between routes.

In L-Space and P-Space, the average values of betweenness $(<\mathrm{BC}>)$ and eigenvector $(<\mathrm{EC}\rangle)$ centralities, the PageRank $<\mathrm{PR}>$, and degree $(<k>)$ of nodes as well as the minimum distance between nodes distributions were estimated. Pagerank was measured considering a damping factor equal to 0.85 . In order to determine if the degree distributions, similar in a way to what happens in other networks [3, 25], followed a power law function, this characteristic was also analysed. Next, all these magnitudes are defined:

(i) The degree of a node $i, k(i)$, for an undirected graph, $G$, such as a PTN, is [26]

$$
k(i)=\sum_{j=1}^{N} A_{i j}
$$

where $A_{i j}$ is the element $i j$ of the adjacency matrix, for example, $A_{i j}=1$ if the node $i$ is linked to node $j$ and 0 otherwise.

(ii) The minimum distance between two nodes $i, j$ in $G$, 1 , is the length of the shortest path between them.

(iii) The betweenness centrality of a node $i$ in $G, \mathrm{BC}(i)$ is [27]

$$
\mathrm{BC}(i)=\sum_{u \neq i \neq w} \frac{\sigma_{u, w}(i)}{\sigma_{u, w}}
$$

where $\sigma_{u, w}$ is the total number of shortest paths from node $u$ to node $w$ and $\sigma_{u, w}(i)$ is the number of those paths that pass through $i$.

(iv) Regarding the eigenvector centrality of a node $i$ in $G$, $\mathrm{EC}(i)[28,29]$ : $\lambda_{1}, \lambda_{2}, \lambda_{3}, \ldots, \lambda_{N}$ are the eigenvalues of the adjacency matrix $A=\left\{A_{i j}\right\}$ of $G$. Then, the largest eigenvalue of matrix $\mathrm{A}$ is $\lambda_{\max }$ with an eigenvector $e=\left[e_{1}, e_{2}, \ldots, e_{N}\right] T$ such that $\lambda_{\max } * e_{i}=\sum_{j=1}^{N} A_{i j}$

$* e_{j}$. The eigenvector centrality for node $i$ denoted as $\mathrm{EC}(i)$ can be defined as

$$
\mathrm{EC}(i)=\frac{1}{\lambda_{\max }} \sum_{j=1}^{N} A_{i j} * e_{j}
$$

(v) Pagerank, PR, of a node $i$ in $G$ is $[28,30]$

$$
\operatorname{PR}(i)=\frac{q}{N}+(1-q) \sum_{j: j \longrightarrow i} \frac{\operatorname{PR}(j)}{k_{\text {out }}(j)}, \quad i=1,2,3, \ldots, N
$$

where $N$ is the number of nodes in $G, \operatorname{PR}(j)$ is the PageRank of a node $j$, and $k_{\text {out }}(j)$ is the outdegree of node $j$. We add $\left(\operatorname{PR}(j) / k_{\text {out }}(j)\right)$ for all nodes with a link ending in $i$. In the case of the PTN, it is considered that $G$ is an undirected graph; therefore, $k_{\text {out }}(j)=k(j) . q$ is the damping parameter, $\in[0,1]$.

Regarding the modularity of the network, in L-Space, this was calculated employing the generate overlapping cluster generator (OCG) method [31]. The clusters were originally hierarchically joined together, optimizing the modularity of the partition, resulting in overlapping clusters. Similarly, to what happens in the protein networks, the uniqueness of node categorization prevents from disclosing the implication of some stops in various trip processes. In L-Space, the assortativity parameter [32] which determines the tendency of nodes being connected to other similar ones was also estimated. Additionally, the Molloy-Reed parameter (MRP) [33] was calculated to know the structural integrity of the networks.

2.4. Simulation of Faults Propagation. In the L-Space, the propagation of failures in each network is simulated using an infection and recovery mechanism, which consists of the following steps:

Calculate the total number of nodes in the network $(N)$, Initialize the infection rate $(\mathrm{IR}=0)$,

Infect the first node,

Calculate the total number of infected nodes in the network $\left(\mu^{\prime}\right)$,

Initialize time $(t=1)$,

while $(\mathrm{IR}<0.60)$ and $(t<T)$ :

\{

Obtain all uninfected nodes in the network, $\left\{\operatorname{uin}_{i}\right\}_{i=1}^{i=\mu}, \mu$ being the total number of uninfected nodes. For $i=1$ to $\mu$

\{ 
Obtain the neighbour nodes of $u i n_{i}\{n n u i$ $\left.n_{i-j}\right\}_{j=1}^{j=\gamma_{i}}, \gamma_{i}$ being the total number of neighbours of $u i n_{i}$ For $j=1$ to $\gamma_{i}$ :

\{ nnuin $_{i-j}$ is still infected

If RandomBinomial $\left(\mu^{\prime}, p I\right)>0$

Else

$$
\text { \} To disinfect } \text { nnuin }_{i-j}
$$

Calculate how many nodes are infected $(\delta)$, For $i=1$ to $\delta$ :

If RandomBinomial then $(1, p R)>0$

then

To disinfect $i n_{i}, p R$ being the recuperation probability,

Else

To infect $i n_{i}$

\}

Calculate the total number of infected nodes in the network $\left(\mu^{\prime}\right)$

Calculate IR as:

$\mathrm{IR}=\mu^{\prime} / N$

\}

where RandomBinomial $(n, p)$ is a generator of random numbers, which are calculated from the binomial distribution specified by the number of trials $n$ and the probability of success for each trial $p$. The np.random.binomial function in python is used.

Several simulations were carried out until a time $T$ and without reaching $60 \%$ percent of the total of infected nodes in each transport network. $p I$ and $p R$ were modified in the range 0 to 0.1 in steps of 0.0025 (values were considered within a range of $0-0.1$, because with $p I>0.1$, all networks very quickly reached $60 \%$ infection (they were saturated in a short time for all groups). It was checked if some differences existed when the propagation process started in nodes with specific characteristics, such as nodes with the highest betweenness (B) and eigenvector centralities (E), nodes with the largest PageRank (P) and degree (D), and nodes randomly selected (A). Pagerank was estimated considering a damping factor equal to 0.85 . Once the simulation was concluded, the existing differences between groups $(\mathrm{B}, \mathrm{E}, \mathrm{P}$, $\mathrm{D}, \mathrm{A})$ were analysed. The following procedure was carried out:

(1) The normality of distributions was tested using the D'Agostino test [34] with a significance level $\alpha=0.05$. This test provides very effective results when it is applied to large size samples [34, 35]. The considered hypothesis was as follows: (i) $H_{0}$ : "the samples came from a normal distribution."

(ii) $H_{\mathrm{a}}$ : "the samples did not come from a normal distribution."

If $p$ value $\leq \alpha, H_{0}$ is rejected and $H_{\mathrm{a}}$ is taken, else $H_{0}$ is accepted.

(2) If normality existed, the homoscedasticity of variances would be studied in each distribution using the Breusch-Pagan test [36] with a significance level of $\alpha=0.05$. The following hypothesis should be taken into account:

(i) $H_{0}$ : "the variance was constant."

(ii) $H_{\mathrm{a}}$ : "the variance was not constant."

Again, if $p$ value $\leq \alpha, \mathrm{H}_{0}$ should be rejected and $H_{\mathrm{a}}$ should be accepted; otherwise, $H_{\mathrm{a}}$ should be taken.

(3) If there was no normality or homoscedasticity in the distributions, the Kruskal-Wallis test [37] should be executed in order to detect whether the population distributions were identical, or at least one differed from the rest. The used significance level was $\alpha=0.05$. The hypothesis employed was as follows:

(i) Null hypothesis: "the groups were from identical populations."

(ii) Alternative hypothesis: "at least one of the groups comes from a different population than the others."

(4) In the next step, the Wilcoxon rank sum test [37] was executed with a significance level $\alpha=0.05$ in order to realize a pairwise comparison between groups. The hypothesis was as follows:

(i) Null hypothesis: "both groups had the same distribution."

(ii) Alternative hypothesis: "both groups have a different distribution."

(5) If there are normality and homoscedasticity, the analysis of variance (ANOVA) method was realized.

2.5. Analysis of Evolution of the GC. The size of the GC describes the largest fraction of overall nodes such that any pair of them is linked through a path. It evaluates the largest extension that a route (or a union of them) provides in terms of the available stops a traveller can reach from an origin inside the GC.

In the L-Space, for each network, the size of GC was calculated as nodes and links were removed according to the following criteria: links (criterion 1) or nodes (criterion 2) randomly eliminated; nodes removed in descending order of betweenness centrality (criterion 3), degree (criterion 4), eigenvector centrality (criterion 5), and PageRank (considering a damping factor equal to 0.85) (criterion 6); and the highest weighted links removed (criterion 7) following a decreasing sequence. In this criterion, a weighted graph was built where the weight of each link, $w_{i j}$, represented the 
number of links between the nodes $i, j$ and the highest number of links between two nodes in the entire network. Finally, the interactive betweenness calculation was carried out (criterion 8). This criterion, instead of removing the nodes in decreasing order of the static betweenness, recomputes the betweenness after the removal of a node.

It is relevant to analyse which of the used criteria allow us to achieve an $80 \%$ reduction in the size of GC in less time. It is true that when the network is fragmented, the stops belonging to the GC are linking a fraction of the PTN, which can still be functional in certain cases. However, it is also relevant to observe the evolution of size over time in order to estimate the attack tolerance. Several commonalities and differences between PTN can be detected. The relevance of studying the evolution of PTN is also corroborated by the interdependencies between PTN and the accessibility to other infrastructures such as hospitals, business centers, schools, and airports. Therefore, the fluctuations in the availability of stops and routes might greatly impact urban life.

\section{Results and Discussion}

3.1. Study of Structural Properties. The PageRank (with a damping parameter equal to 0.85), betweenness and eigenvector centralities, degree, and minimum distance characteristics were analysed in the L-Space and P-Space. Figures 1 and 2 show the L-Space for all studied networks. The average values of degree, PageRank, eigenvector, and betweenness centralities for this space can be observed in Table 1. Those correspond to degrees, which are similar in all networks. The average eigenvector and PageRank centralities are lower than 0.002 , having very small values in all networks. Therefore, the failures randomly happening in a node will have a low probability of arriving at any other. The same happens with respect to betweenness centrality, presenting small magnitudes (a mean value minor than 0.025), which denotes a slight average in how a node plays a bridging role in a network. Table 1 also shows the value of MRP in all networks, proving the existence in them of a GC $($ MRP $>2)$.

In L-Space, an equivalence between the degree distributions of the PTN of some cities was pointed out by Von Feber et al. [3], where the authors showed that this distribution followed a power law. This characteristic was checked for the analysed networks, fitting their degree distribution to the function:

$$
P(k) \sim k^{-\alpha} .
$$

The obtained values for the fitting are shown in Table 2. It is observed, according to the $\mathrm{R}$ square value, that those obtained for the fitting were not good ( $R$ squared is not close to $1)$. Figure 3 depicts, in L-Space, the degree distribution for the analysed networks. It is clear that all of them have a low value of $k\left(k_{\max } \leq 17\right)$ compared with the total links. Therefore, the failures occurring at random on a node will have a high probability of having a small impact. Even so, these networks might be vulnerable to disruptions since a very high amount of nodes is joined to very few other nodes (low degree).
It is clear from the above that the values of betweenness, eigenvector, and PageRank centralities in the analysed networks ensure that they are highly robust against random failures.

The lowest average minimum distance between nodes shows a relevant variability in all networks. TransAntofagasta and EMT as well as AVL, CFL, RGTR, and TICE networks have the lowest value. The highest value corresponds to Thunder Bay Transit network.

The modularity of the network is also evaluated in L-Space, employing the generate overlapping cluster generator (OCG) method [31]. It can be noted in Table 3 that all networks show a high value of $Q$ with magnitudes near to 1 , which demonstrates a strong community structure in these networks. Romano et al. [38] showed that network efficiency is modularity dependent, with the highest values of transmission occurring at intermediate levels and low values of modularity. High values tend to negatively influence transmission in networks [38]. Therefore, the high modularity in PTN could help to slow down the propagation of a failure in the network.

Table 4 depicts the values of the assortativity coefficient [32]. All networks show assortative higher than 0.4, demonstrating that a significant chance exists in which fractions of stops will randomly join with stations of the same degree. The nodes appear to join with a specific preference. A failure in a node with the highest degree will not have much negative impact, since other nodes with the highest degree are still connected to ones with a similar degree.

The highest $k$-core in all networks was 3 or 2 , demonstrating that the largest subgraphs exist where every node is connected to at least 3 or 2 other nodes within the network. Most nodes show a small and similar $k$-core $(\leq 2)$.

With respect to P-Space, Table 5 shows the average values of eigenvector and betweenness centralities, PageRank, degree, minimum distance between nodes, diameter, and density.

The degree distributions of the analysed networks were also fitted to a power law function according to equation (5). The results are shown in Table 6. It can be noted that, as in the case of L-Space, in accordance with the obtained $R$ square value, the fitting is not good. P-Space allows us to analyse interconnections between routes and transfers in the network. In this space, $k$ reveals how many stops a person can travel through by using only one route. The network with the highest value average $k,\langle k\rangle$, is TransAntofagasta. The obtained values for eigenvector and PageRank centralities are very low and similar in all networks, reinforcing the idea exposed in the analysis of L-space that failures randomly occurring in a node have a low probability of quickly arriving at any other one. Many possible transfer nodes exist, making the values for betweenness centrality very small and achieving high robustness against random failures on a node. The PTNs with lower $\langle l\rangle$ are the best connected in this space, having a minor level of vulnerability. Figure 4 shows, in P-Space, the degree distribution for the analysed networks. 


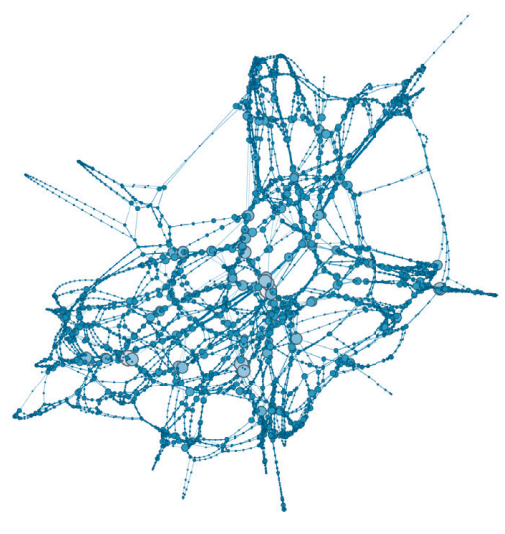

(a)

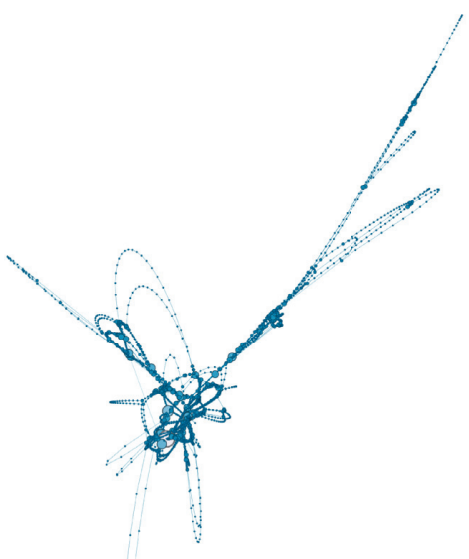

(b)

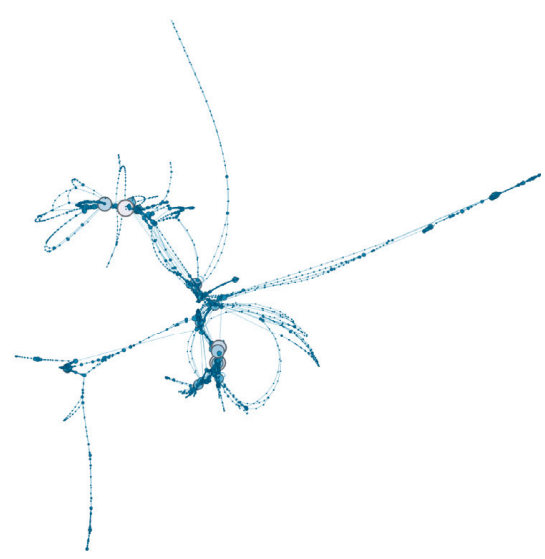

(c)

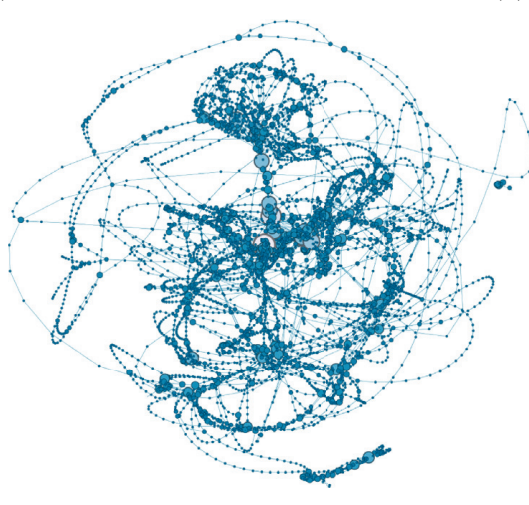

(d)

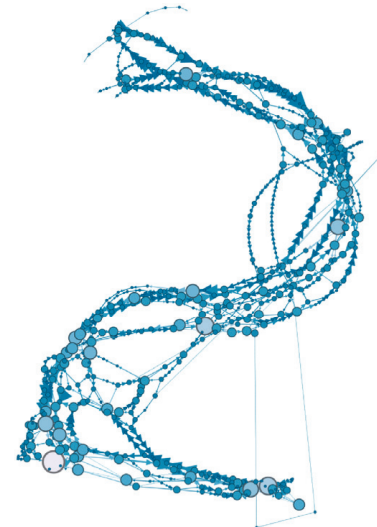

(e)

Figure 1: In L-Space, for EMT (a), BC Transit (b), Kolumbus (c), Auckland (d), and TransAntofagasta (e) networks.

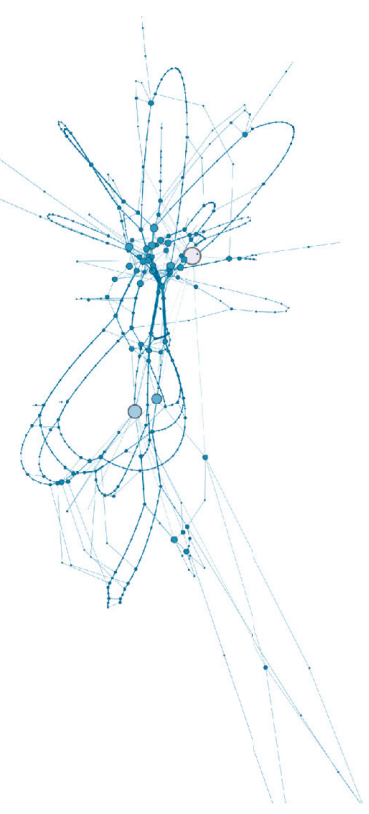

(a)

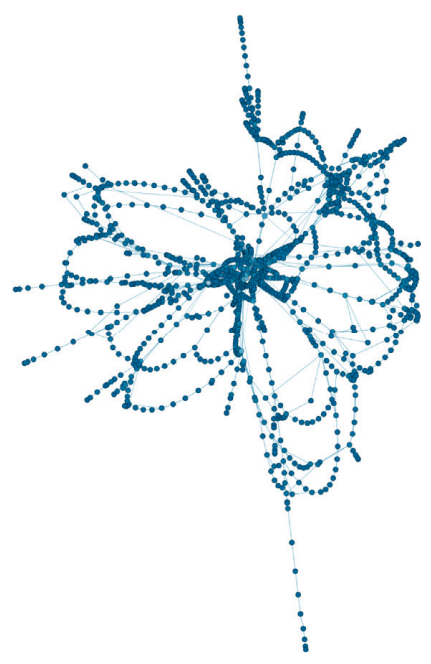

(b)

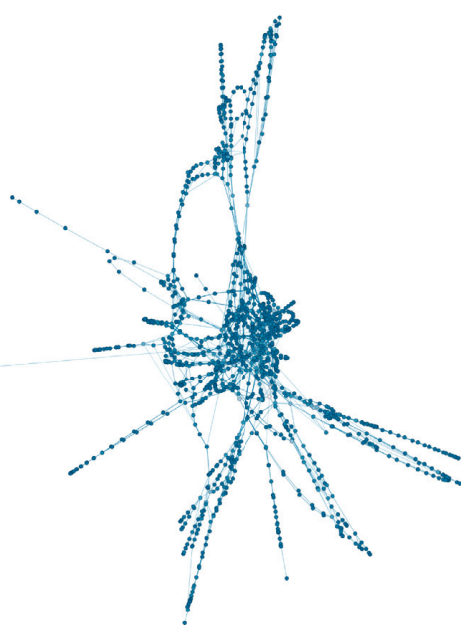

(c)

Figure 2: Continued. 


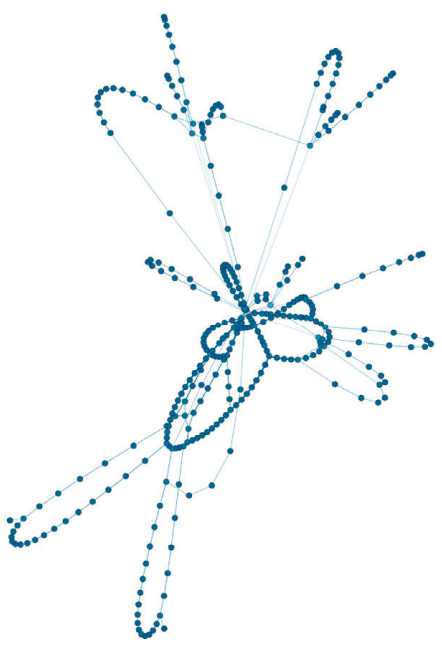

(d)

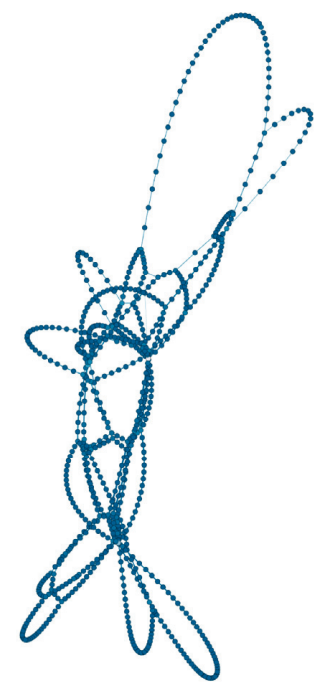

(e)

Figure 2: In L-Space, for Linja-Karjala Oy (a), AVL, CFL, RGTR, and TICE (b), STAR (c), CIT (d), and Thunder Bay Transit (e) networks.

TABLE 1: Structural properties in L-Space. Average Betweenness Centrality $(<\mathrm{BC}>)$, average degree $(<k>)$, average Eigenvector Centrality $(<\mathrm{EC}>)$, average PageRank (with a damping factor $=0.85)(<\mathrm{PR}>)$, average path Length $(<l>)$, diameter $(d)$, density, and MRP.

\begin{tabular}{lccccccccccc}
\hline Network & $\begin{array}{c}\text { Number of } \\
\text { nodes }\end{array}$ & $\begin{array}{c}\text { Number of } \\
\text { links }\end{array}$ & $\langle$ BC $>$ & $<k>$ & $\langle$ EC $>$ & $<$ PR $>$ & $<l>$ & $d$ & Density & MRP \\
\hline Auckland & 5,223 & 318,896 & 0.00655 & 2.36071 & 0.00217 & 0.00019 & 2.69523 & 151 & 0.00045 & 2.66878 \\
EMT & 4,636 & $2,070,508$ & 0.00383 & 2.71140 & 0.00181 & 0.00022 & 1.00000 & 61 & 0.00058 & 3.21782 \\
BC Transit & 3,981 & 702,197 & 0.00233 & 2.28894 & 0.00167 & 0.00025 & 9.11427 & 83 & 0.00058 & 2.54653 \\
Kolumbus & 3,829 & 487,432 & 0.00258 & 2.59546 & 0.00216 & 0.00026 & 2.89389 & 98 & 0.00068 & 3.24314 \\
STAR & 1,415 & 661,150 & 0.00664 & 2.81696 & 0.00559 & 0.00071 & 10.0000 & 37 & 0.00199 & 3.54340 \\
AVL, CFL, RGTR, and TICE & 1,372 & 340,684 & 0.00706 & 2.81050 & 0.00564 & 0.00072 & 1.00000 & 34 & 0.00205 & 3.79201 \\
Thunder Bay Transit & 825 & 78,247 & 0.03499 & 2.17697 & 0.00560 & 0.00121 & 29.79 & 92 & 0.00264 & 2.35412 \\
TransAntofagasta & 650 & 724,362 & 0.02436 & 2.96308 & 0.00902 & 0.00154 & 16.78525 & 51 & 0.00456 & 3.46729 \\
Linja-Karjala Oy & 551 & 63,399 & 0.01611 & 2.55898 & 0.01497 & 0.00181 & 4.50476 & 29 & 0.00465 & 3.18865 \\
CIT & 346 & 9,366 & 0.02714 & 2.24855 & 0.01700 & 0.00289 & 2.33333 & 29 & 0.00651 & 2.75804 \\
\hline
\end{tabular}

TABLE 2: Fitting in L-Space of the network degree distribution to a power law function $P(k) \sim k^{-\alpha}$.

\begin{tabular}{lcc}
\hline & \multicolumn{2}{c}{ L-Space } \\
Network & $\alpha$ & $R$ square \\
\hline Linja-Karjala Oy & 0.158 & 0.600 \\
Kolumbus & 0.459 & 0.330 \\
EMT & 0.622 & 0.239 \\
Auckland & 0.603 & 0.237 \\
BC Transit & 0.412 & 0.161 \\
AVL, CFL, RGTR, and TICE & 0.236 & 0.156 \\
CIT & 0.302 & 0.156 \\
Thunder Bay Transit & 0.406 & 0.068 \\
TransAntofagasta & 0.182 & 0.044 \\
STAR & 0.158 & 0.041 \\
\hline
\end{tabular}

3.2. Simulation of Faults Propagation. Failure propagation was simulated in L-Space using an infection and recovery algorithm.
(1) Firstly, a total of 100 experiments for each selection criterion and duple $(p I, p R)$ were undertaken in each network. In each experiment, the simulation was implemented until $t=T$ or until the percentage of infected nodes was higher or equal to $60 \%$. The values of $t$ were modified in steps of 10 units.

(2) The second step was to check the normality of distributions of the infection rate (IR), in all groups (A, B, D, E, P) using the D'Agostino test for any T, which took different values until reaching a level propagation $\geq 60 \%$ of the network. The results show that $p$ value was not higher than 0.05 in all cases. Since not all distributions presented normality, it was not necessary to study the homoscedasticity of variances applying the Breusch-Pagan Test. The Kruskal-Wallis test could be used for the detection of differences between groups.

(3) The third step was using the Kruskal-Wallis test to verify for each $T$, whether all groups $(A, B, D, E, P)$ were identical or by contrast, one of the groups 


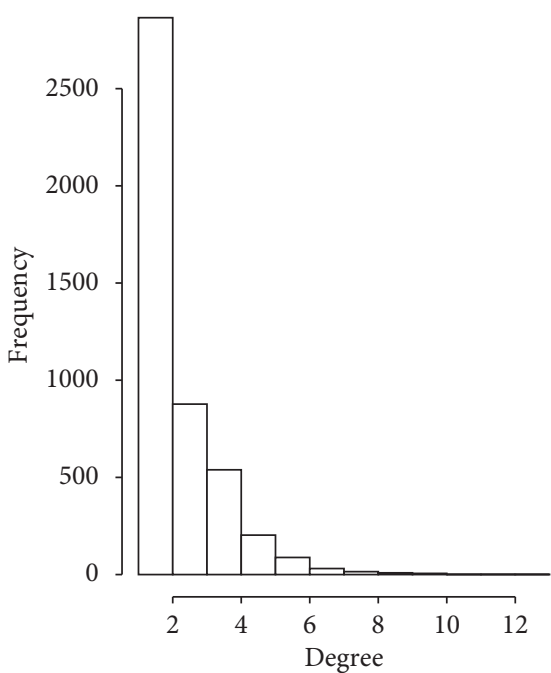

(a)

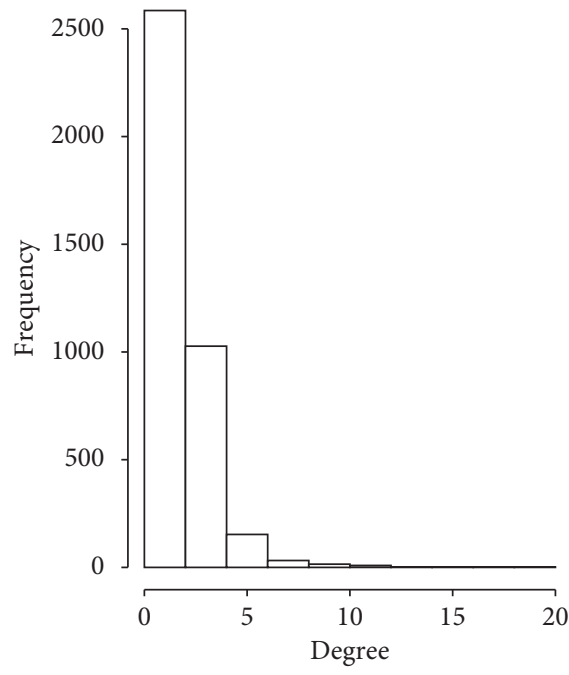

(c)

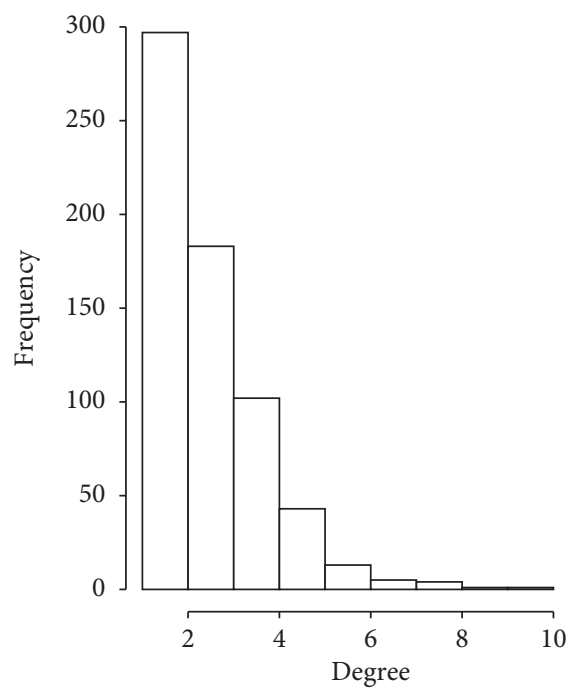

(e)

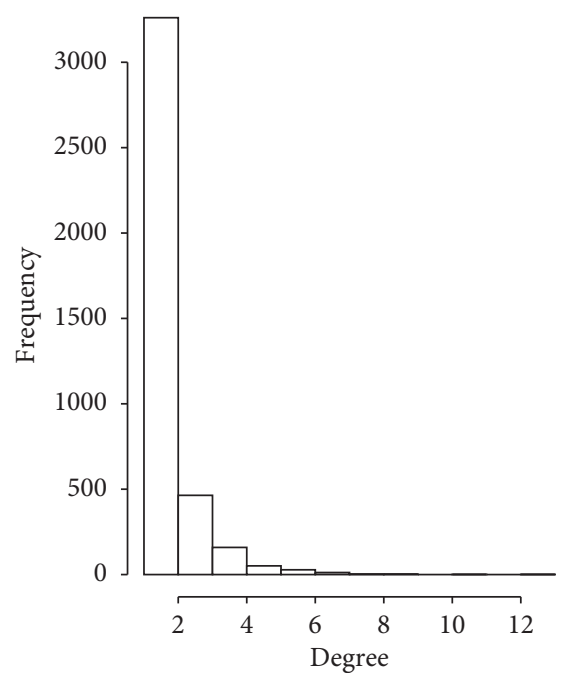

(b)

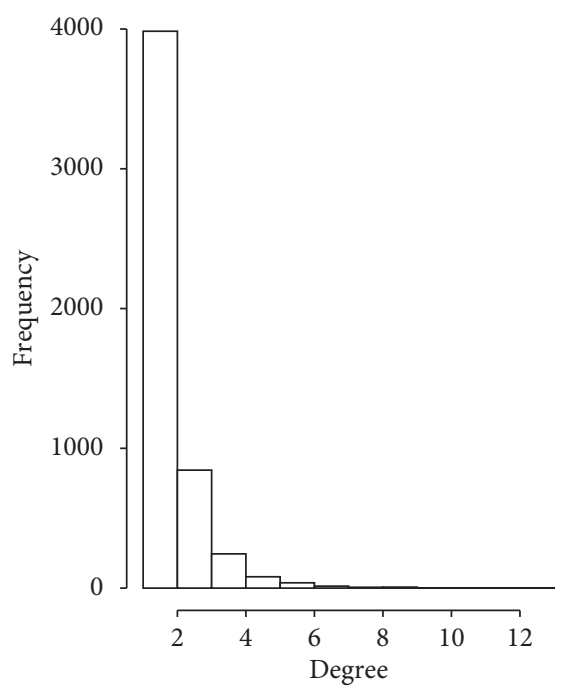

(d)

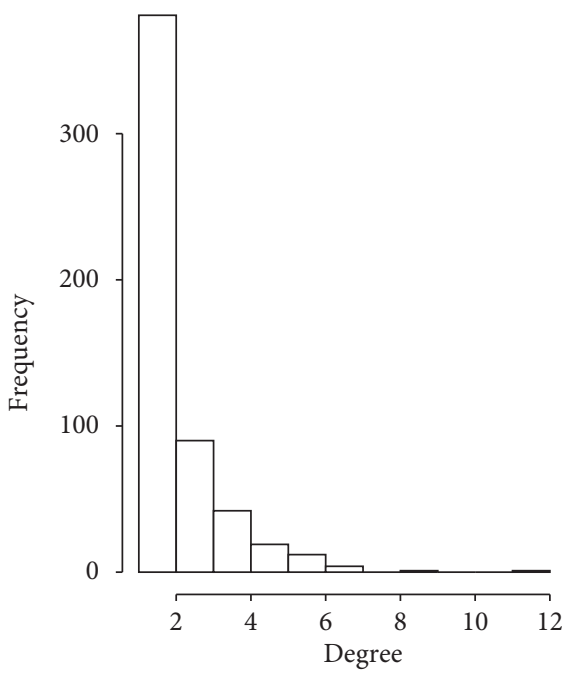

(f)

Figure 3: Continued. 


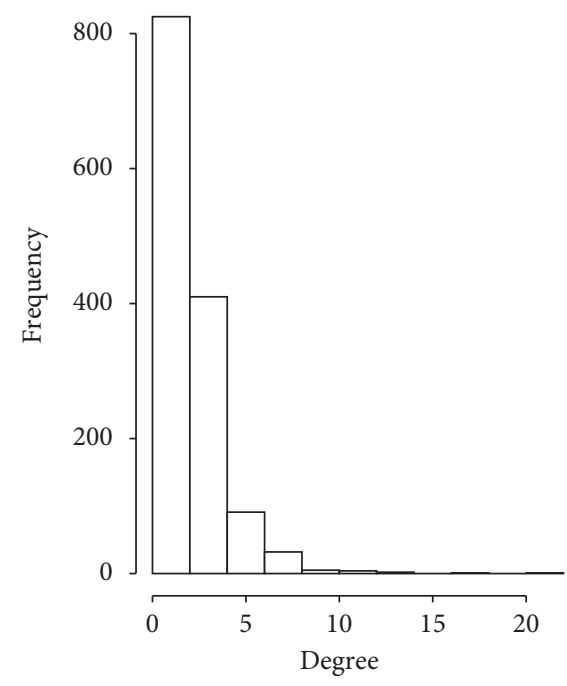

(g)

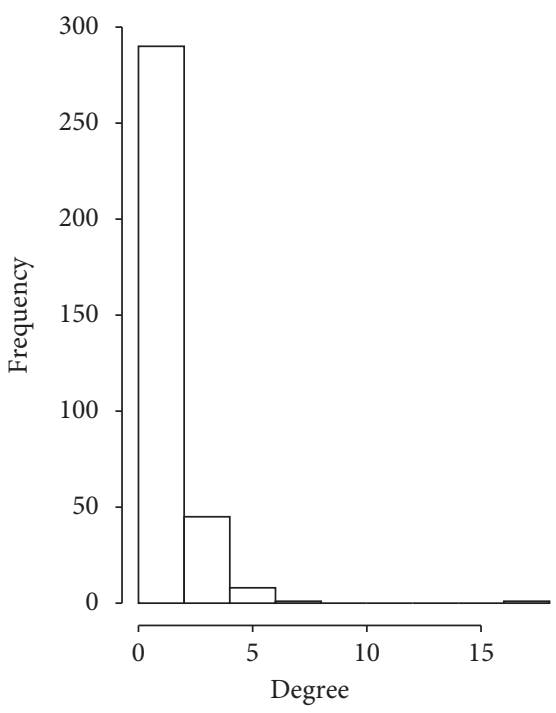

(i)

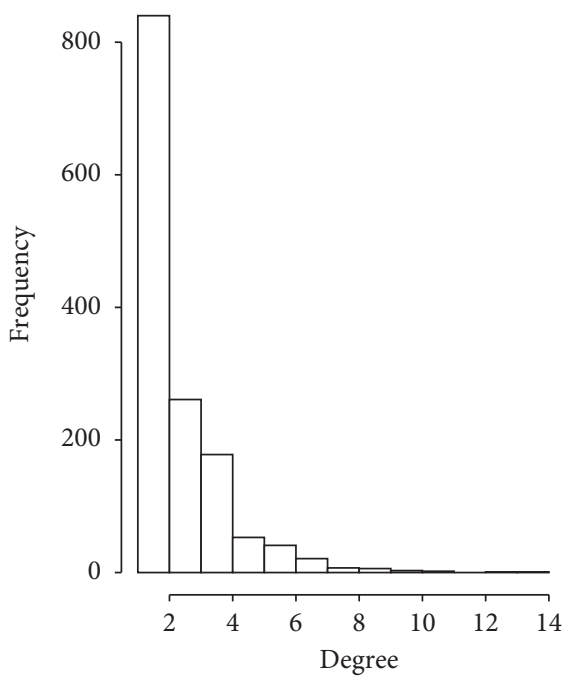

(h)

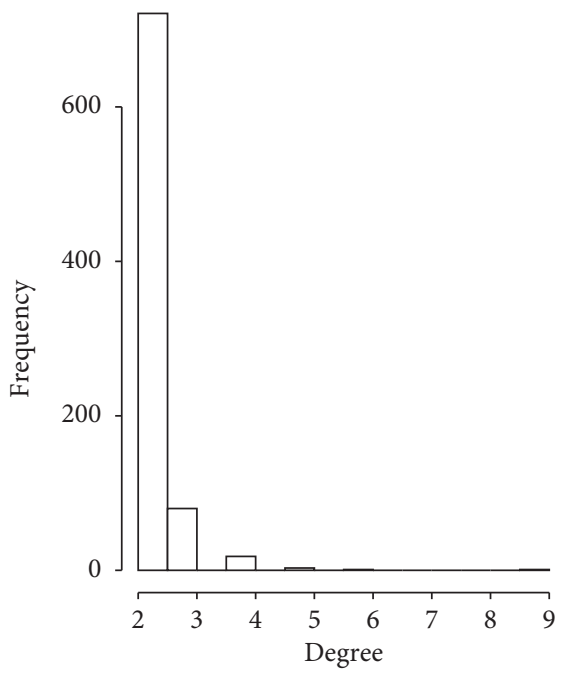

(j)

Figure 3: Degree distribution in linear ( $x$-axis) and linear scale ( $y$-axis) for the following networks: EMT (a), BC Transit (b), Kolumbus (c), Auckland (d), TransAntofagasta (e), Linja-Karjala Oy (f), AVL, CFL, RGTR, TICE (g), STAR (h), CIT (i), and Thunder Bay Transit (j).

TABLe 3: $Q$ Modularity computed using the overlapping cluster generator (OCG) method in L-Space.

\begin{tabular}{lc}
\hline Network & $Q$ \\
\hline BC Transit & 0.99750 \\
Auckland & 0.99610 \\
EMT & 0.98550 \\
Thunder Bay Transit & 0.99100 \\
Kolumbus & 0.98730 \\
STAR & 0.97020 \\
AVL, CFL, RGTR, and TICE & 0.96880 \\
CIT & 0.95880 \\
TransAntofagasta & 0.93910 \\
Linja-Karjala Oy & 0.93960 \\
\hline
\end{tabular}

tended to give observations that were different from those of other groups. Again, the $p$ value obtained was lower than 0.05 . The null hypothesis had to be rejected because at least one group had a different distribution.

(4) The fourth step was carried out utilising the Wilcoxon rank sum test in order to compare paired groups; we also applied the Bonferroni correction, both with a significance level $\alpha=0.05$.

In all analysed duos $(p I, p R)$, for EMT, there was a significant difference ( $p$ value $\leq 0.05$ ) between all groups except between groups $\mathrm{D}-\mathrm{E}$. With respect to BC Transit network, there were dissimilarities $(p$ value $\leq 0.05)$ between some groups except between groups D-E, D-P, and E-P. Regarding the Kolumbus network, a relevant similarity is found between groups $\mathrm{B}-\mathrm{E}$ and $\mathrm{P}-\mathrm{D}$. In the Auckland network, similarities happened between groups $\mathrm{P}-\mathrm{D}, \mathrm{P}-\mathrm{B}$, and $\mathrm{D}-\mathrm{B}$. For $p I=0.05$ and $p R=0.0025$, in $T=100$, Figure 5 shows the results of the propagation 
TABLE 4: Assortativity coefficients in L-Space.

\begin{tabular}{lc}
\hline Network & Assortativity \\
\hline Kolumbus & 0.90599 \\
STAR & 0.89463 \\
BC Transit & 0.86067 \\
Auckland & 0.84123 \\
Linja-Karjala Oy & 0.81336 \\
AVL, CFL, RGTR, and TICE & 0.72469 \\
EMT & 0.67211 \\
TransAntofagasta & 0.58252 \\
Thunder Bay Transit & 0.45575 \\
CIT & 0.42243 \\
\hline
\end{tabular}

algorithm in EMT, BC Transit, Kolumbus, and Auckland networks. For the same values of $p I$ and $p R$ and $T=65$, Figure 6 shows the outcomes for STAR and AVL, CFL, RGTR, and TICE networks. Figure 7 depicts the results for TransAntofagasta, Linja-Karjala Oy, CIT, and Thunder Bay Transit networks. For those values of $p I, p R$, and $T$, Tables 7-16 show the obtained $p$ value in the Wilcoxon rank sum test in each network.

For STAR networks, in all studied duos $(p I, p R)$ there was a similarity ( $p$ value $>0.05$ ) between groups $\mathrm{B}-\mathrm{D}, \mathrm{B}-\mathrm{E}$, $\mathrm{B}-\mathrm{P}$, and D-P. In AVL, CFL, RGTR, and TICE network there is an equivalence between groups $\mathrm{E}-\mathrm{D}$ and $\mathrm{B}-\mathrm{P}$.

With respect to the TransAntofagasta network, a similarity exists between groups D-P. In Linja-Karjala Oy and CIF networks, there is a difference between groups $A-B$, A-D, A-E, and E-P. Additionally, dissimilarities exist between B-E groups in the Thunder Bay Transit network.

In all analysed networks, according to the analysis carried out, the A category presents differences with respect to other groups, showing that those failures randomly happening on a node have a distinct behaviour to those originated by target attacks.

In all networks, for $p I=0.05$ and $p R=0.0025$, Tables 17 and 18 show the time in which IR reaches $60 \%$.

It can be noted that in all analysed networks, random failures take the longest propagation time, demonstrating, as was previously covered in the section labelled study of structural properties, that these networks are less vulnerable to such failures. Depending on the network, some categories (D, E, P, or B) show similar propagation times. This seems to point out that in some cases, there is an existence of a certain amount of correlation between betweenness, degree, PageRank, and eigenvector centralities. Research shows that topology and density significantly affect the correlation between centrality measures and suggested that they could also affect the robustness [39].

The networks that showed reduced failure propagation times were AVL, CFL, RGTR, and TICE; STAR; LinjaKarjala Oy; and TransAntofagasta. However, those that presented the highest times were in the order of BC Transit, Kolumbus, Auckland, EMT, and finally Thunder Bay Transit. In the magnitude of these times, there is an influence of the diameter presented by the network in L-space.
3.3. Analysis of Evolution of the GC. It is necessary to know the sensitivity of the network to the elimination of stops and links according to certain criteria in order to investigate which of them has a higher impact. For criteria 1-7, the nodes are removed in blocks of five elements and the percentage of the reduction in the GC is calculated. The applied criteria are the removal of random links (criterion 1) and the removal of random nodes (criterion 2). Other strategies such as the removal of nodes in the descending order of betweenness centrality (criterion 3), degree (criterion 4), eigenvector centrality (criterion 5), PageRank (with a damping factor equal to 0.85) (criterion 6), and highest weight links (criterion 7) are also applied. Using this criterion, a weighted graph was built, in which the weight of each link, $w_{i j}$, represented the portion of links taken from the total number of links between $i$ and $j$. The interactive betweenness calculation (criterion 8) was also used. Figures 8 and 9 show, in all networks, the obtained results for the eight criteria.

In Table 19 and Figures 8 and 9, it can be observed that in all networks, the four criteria that most quickly achieve $80 \%$ of the reduction of the GC after removing a number of nodes in the range $[0 \%, 20 \%]$ have the highest interactive betweenness, PageRank, degree, and static betweenness centrality. And among them, in concordance with [6], in most networks, the elimination of nodes with the highest betweenness interactively calculated obtained an $80 \%$ reduction in the GC more rapidly, with a minor \% of nodes eliminated. The criteria in which $80 \%$ was reached more slowly were those with a removal of random links, random nodes, the highest weighted links, and eigenvector centrality.

The information above highlights the importance of taking special protective measures at some stops. The networks are more robust to failures in random links, random nodes, the highest weighted links, and eigenvector centrality than in the nodes with the highest interactive betweenness, PageRank, static betweenness centrality, and degree. However, when the faults occur randomly, those happening in links produce a higher impact. There is consistency with [40], which proves that links are more robust than nodes when the problems happen in highest degree nodes or in links, for instance, with a weight based on passengers' flow between stops. 
TABle 5: Structural properties in P-Space. Average Betweenness Centrality $(<\mathrm{BC}>)$, average degree $(<k>)$, average Eigenvector Centrality $(<\mathrm{EC}>)$, average PageRank (with a damping factor $=0.85)(<\mathrm{PR}>)$, average path Length $(<l>)$, diameter $(d)$, and density.

\begin{tabular}{|c|c|c|c|c|c|c|c|c|c|}
\hline Network & Number of nodes & Number of links & $<\mathrm{BC}>$ & $<k>$ & $<\mathrm{EC}>$ & $<\mathrm{PR}>$ & $<l>$ & $d$ & Density \\
\hline Auckland & 5,223 & $8,079,095$ & 0.00039 & 101.28508 & 0.02386 & 0.00019 & 2.69143 & 7 & 0.01940 \\
\hline EMT & 4,010 & $25,263,108$ & 0.00065 & 47.66983 & 0.04685 & 0.00025 & 3.59238 & 7 & 0.01189 \\
\hline BC Transit & 3,981 & $15,737,729$ & 0.00017 & 70.76714 & 0.03415 & 0.00025 & 2.65481 & 6 & 0.01778 \\
\hline Kolumbus & 3,829 & $11,317,714$ & 0.00028 & 78.98355 & 0.02584 & 0.00026 & 2.96889 & 7 & 0.02063 \\
\hline STAR & 1,415 & $9,477,213$ & 0.00137 & 43.11236 & 0.01862 & 0.00071 & 1.48276 & 6 & 0.03049 \\
\hline AVL, CFL, RGTR, and TICE & 1,372 & 4,25 & 0.00120 & 48.42570 & 0.01791 & 0.00073 & 1.00000 & 6 & 0.03532 \\
\hline Thunder Bay Transit & 825 & $2,014,779$ & 0.00133 & 75.51030 & 0.02197 & 0.00121 & 2.09 & 4 & 0.09164 \\
\hline TransAntofagasta & 650 & $31,171,307$ & 0.00108 & 199.74769 & 0.03666 & 0.00154 & 1.70123 & 3 & 0.30778 \\
\hline Linja-Karjala Oy & 551 & $1,167,682$ & 0.00180 & 64.26860 & 0.03500 & 0.00181 & 1.47619 & 4 & 0.11685 \\
\hline CIT & 346 & 135,740 & 0.00274 & 33.93478 & 0.03445 & 0.00289 & 1.00000 & 4 & 0.103578 \\
\hline
\end{tabular}

TABle 6: Fitting in P-Space of the network degree distribution to a power law function.

\begin{tabular}{lcc}
\hline Network & $\alpha$ & $R$ square \\
\hline EMT & 0.513 & 0.180 \\
Kolumbus & 0.169 & 0.166 \\
Auckland & 0.204 & 0.077 \\
BC Transit & 0.177 & 0.068 \\
Thunder Bay Transit & 0.275 & 0.047 \\
TransAntofagasta & 0.163 & 0.044 \\
STAR & 0.118 & 0.037 \\
CIT & 0.109 & 0.036 \\
AVL, CFL, RGTR, and TICE & 0.061 & 0.022 \\
Linja-Karjala Oy & 0.015 & 0.001 \\
\hline
\end{tabular}

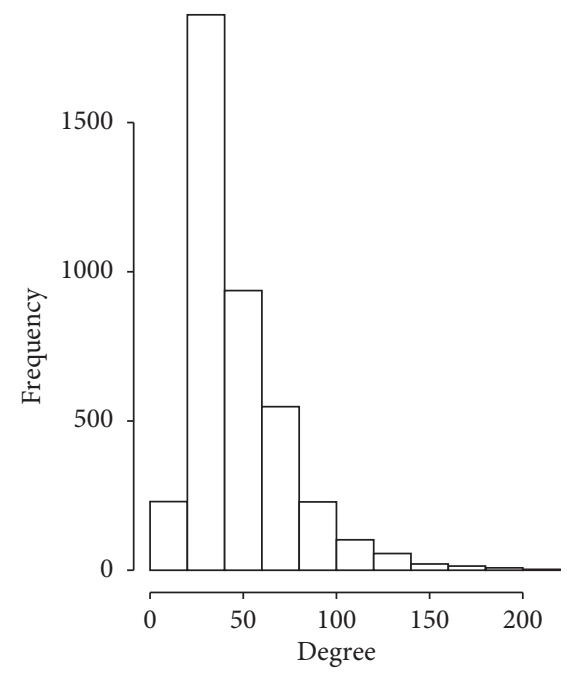

(a)

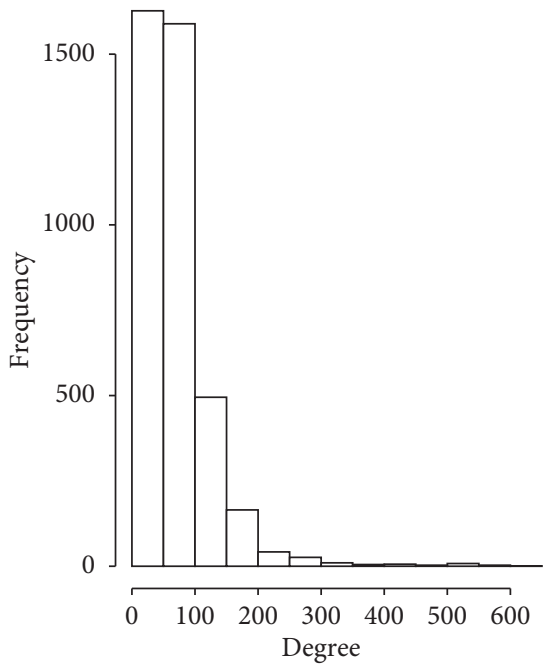

(b)

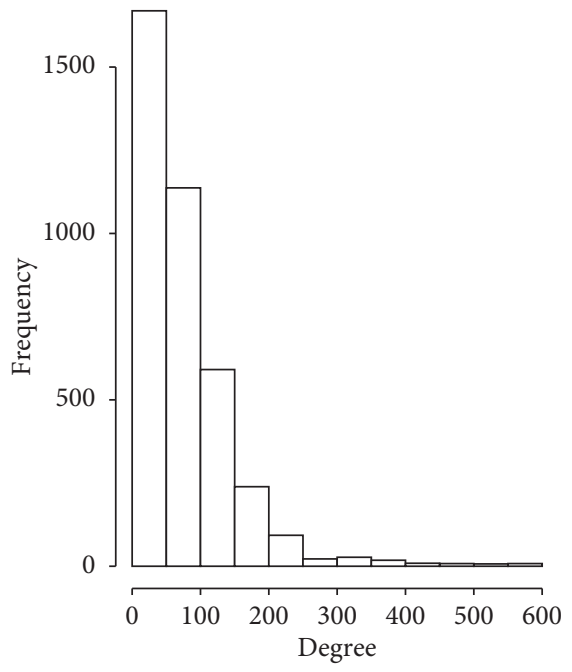

(c)

Figure 4: Continued. 


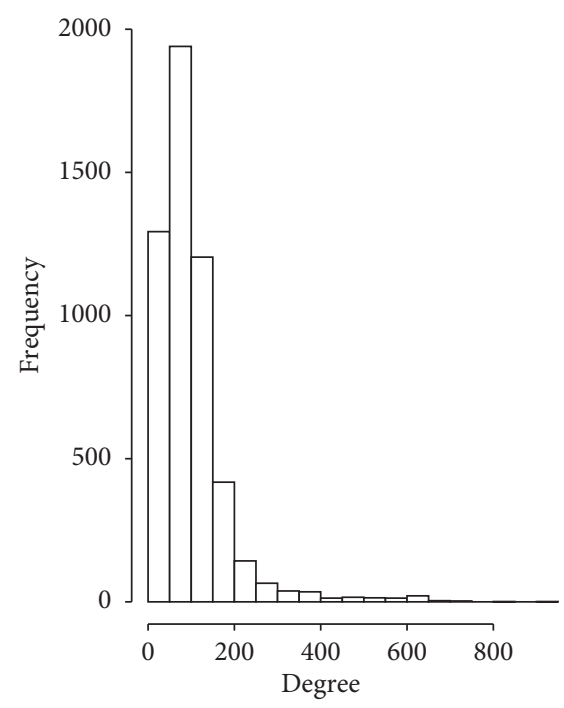

(d)

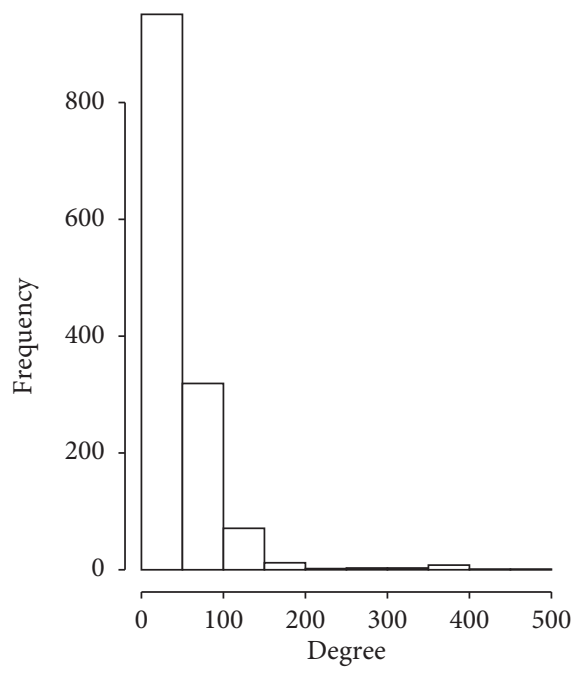

(g)

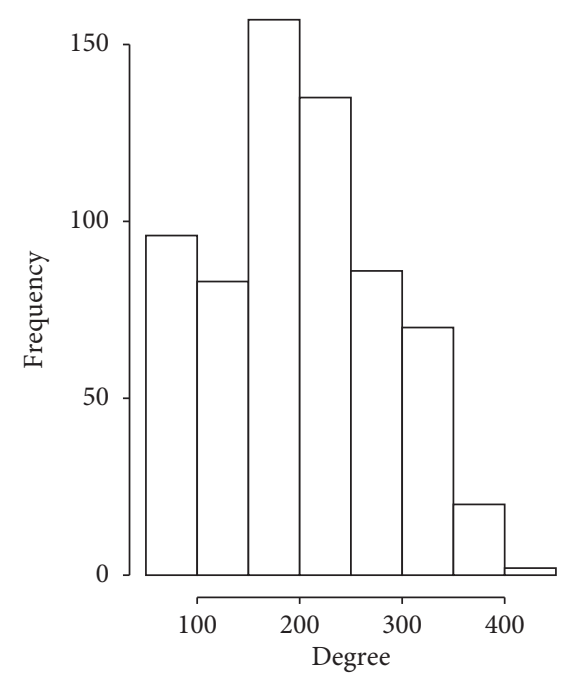

(e)

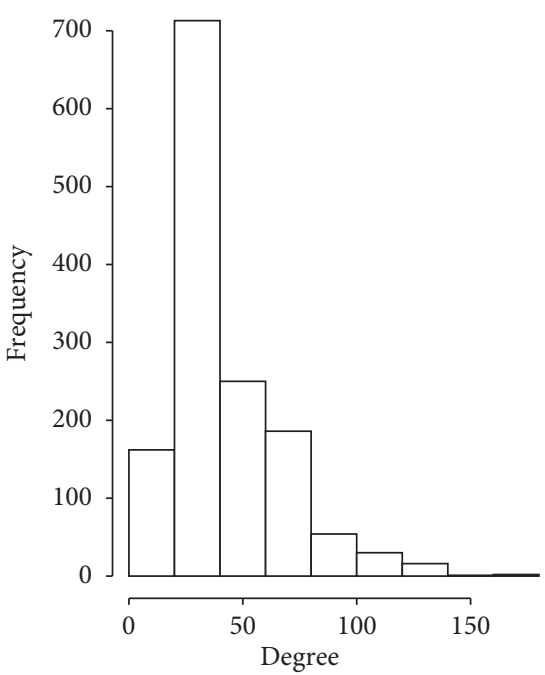

(h)

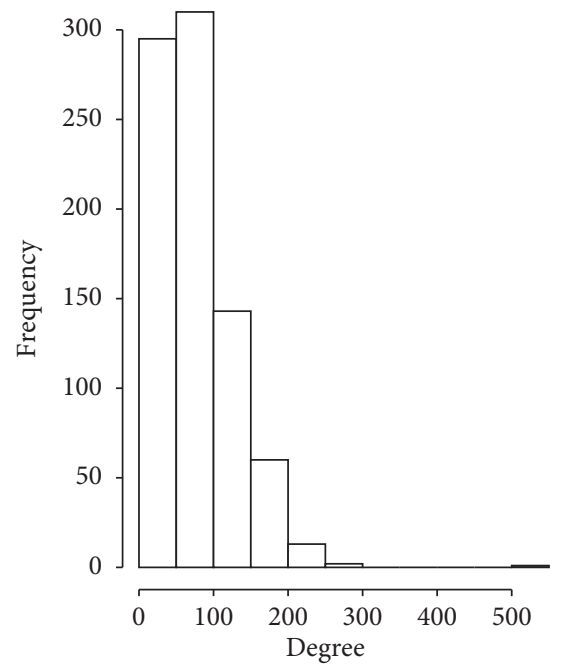

(j)

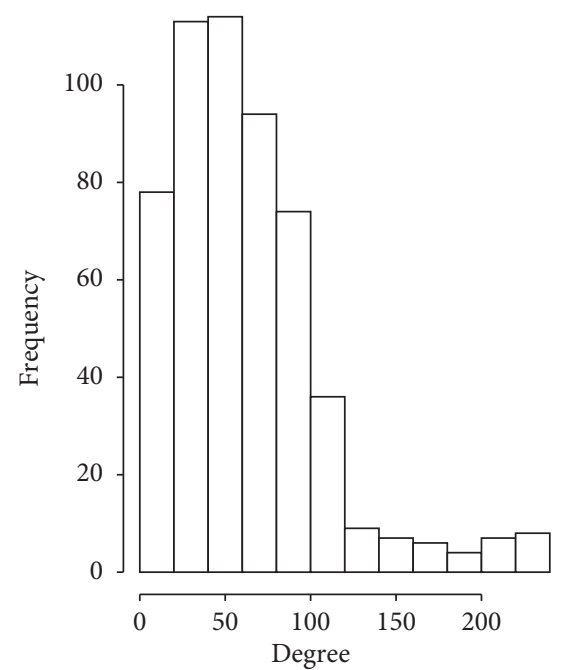

(f)

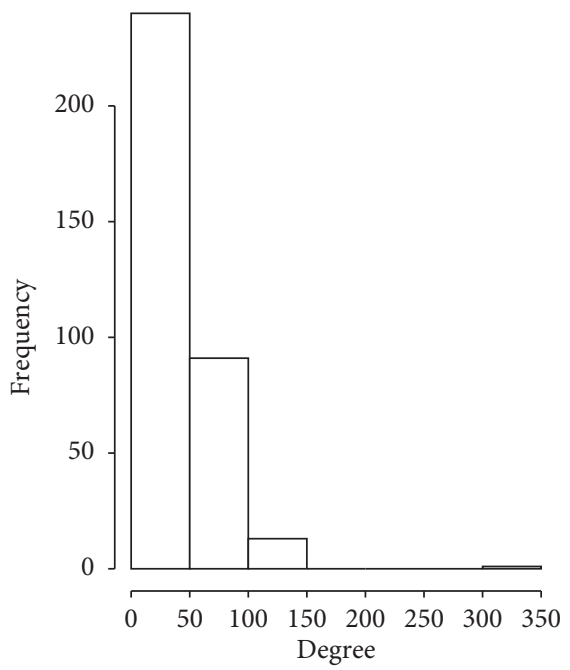

(i) Kolumbus (c), Auckland (d), TransAntofagasta (e), Linja-Karjala Oy (f), AVL, CFL, RGTR, and TICE (g), STAR (h), CIT (i), and Thunder Bay Transit (j) 


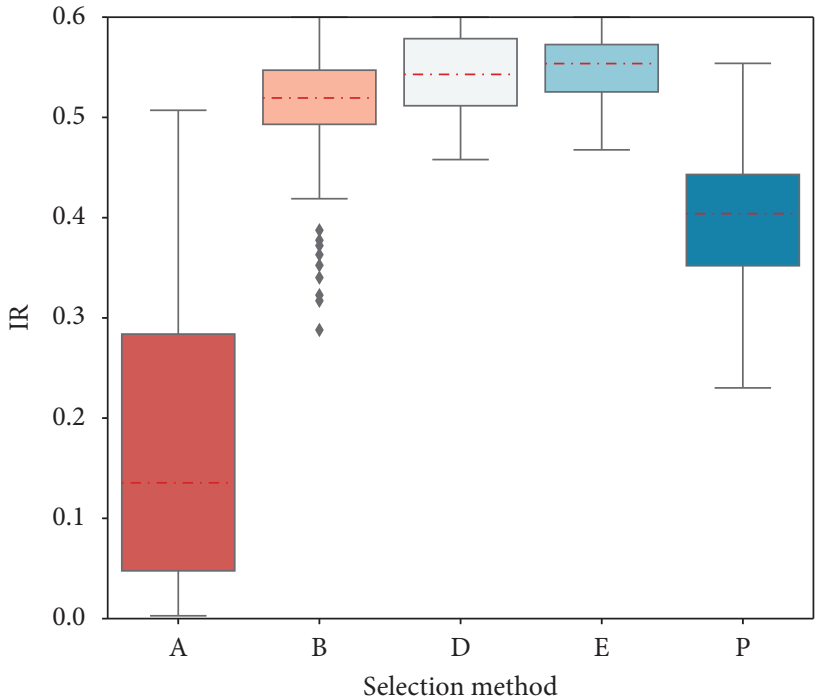

(a)

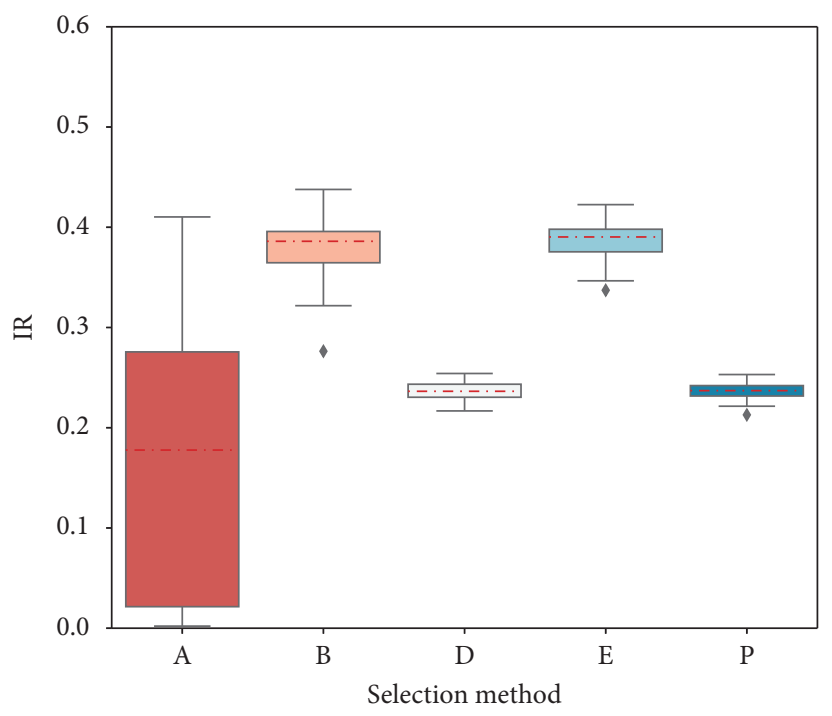

(c)

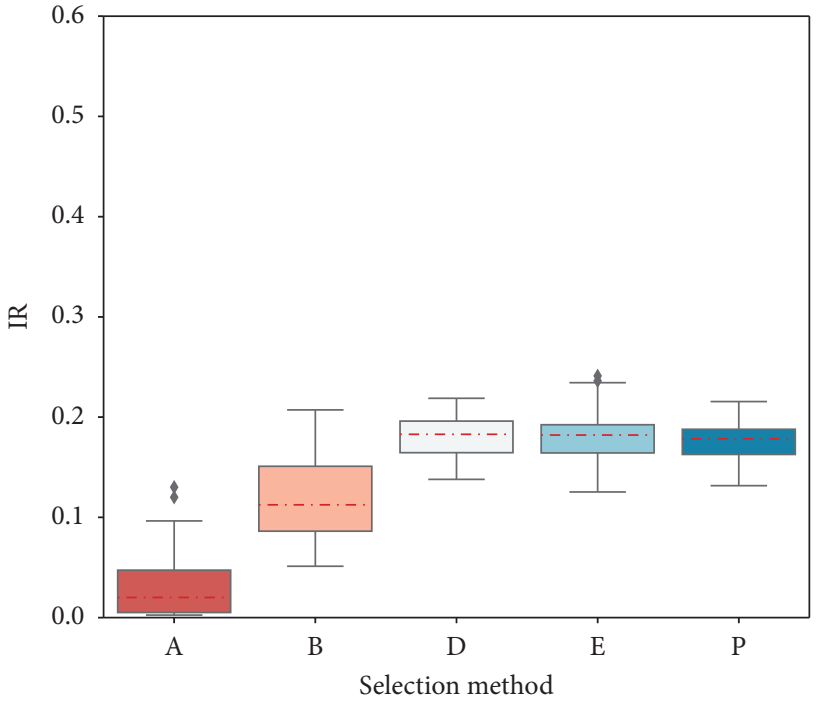

(b)

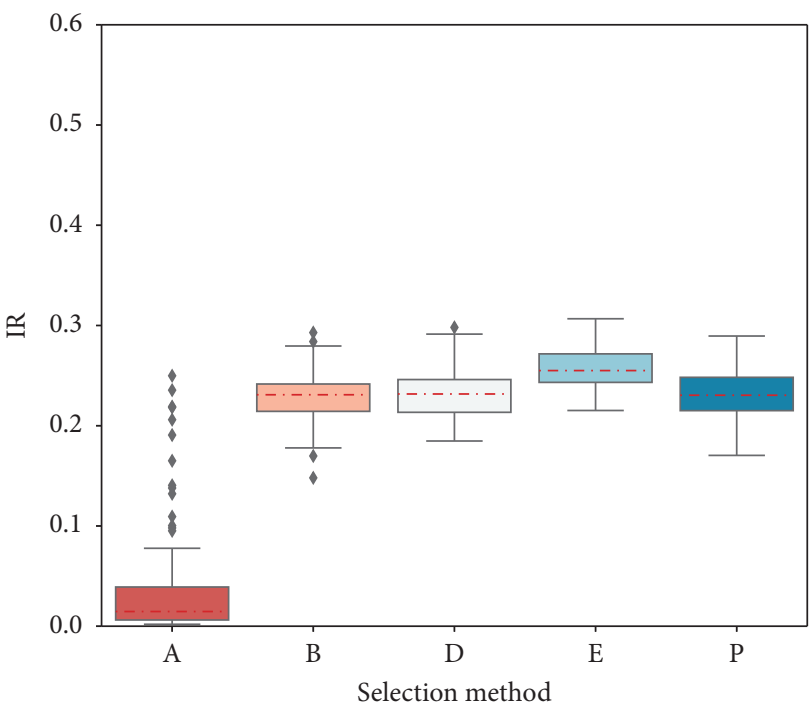

(d)

Figure 5: In L-Space for (a) EMT, (b) BC Transit, (c) Kolumbus, and (d) Auckland networks. $p I=0.05, p R=0.0025$, and $T=150$ (boxplot diagram for $\mathrm{A}, \mathrm{B}, \mathrm{D}, \mathrm{E}$, and $\mathrm{P}$ groups).

Regarding $k$-core feature, it does not seem to influence the level of impact that a failure originating from a node will have on the network. This is in line with some research points, in reference to social networks, where in rumour spreading models, only the $k$-core of a node does not determine its propagation capacity [41].
Additionally, if some of the topological parameters in L-Space, which are depicted in Table 1, are seen in conjunction with the results shown in Tables 17 and 18, it can be noted that the networks with the highest value of density and lowest value of diameter are between those that obtain $60 \%$ in the IR more quickly. 


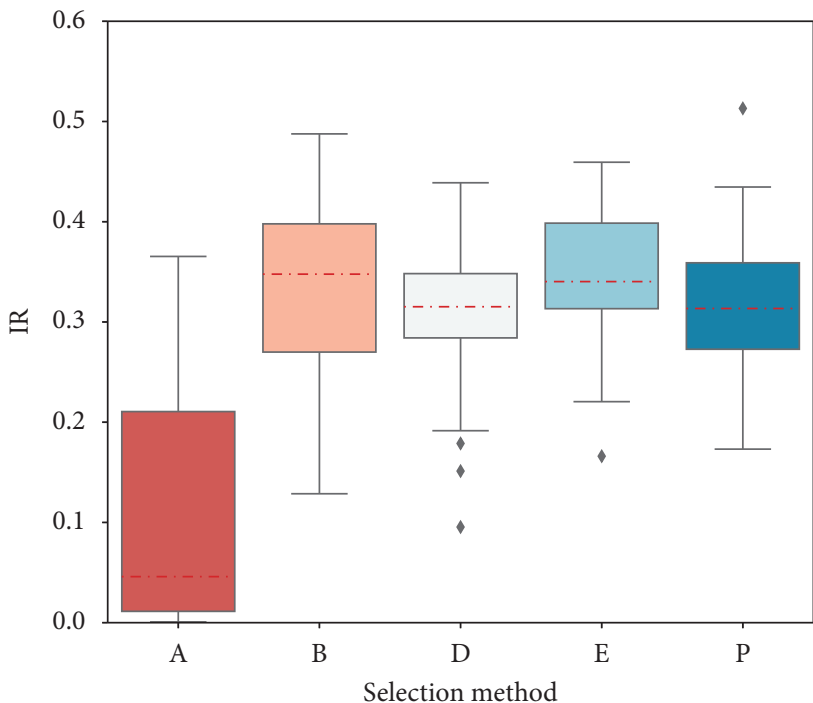

(a)

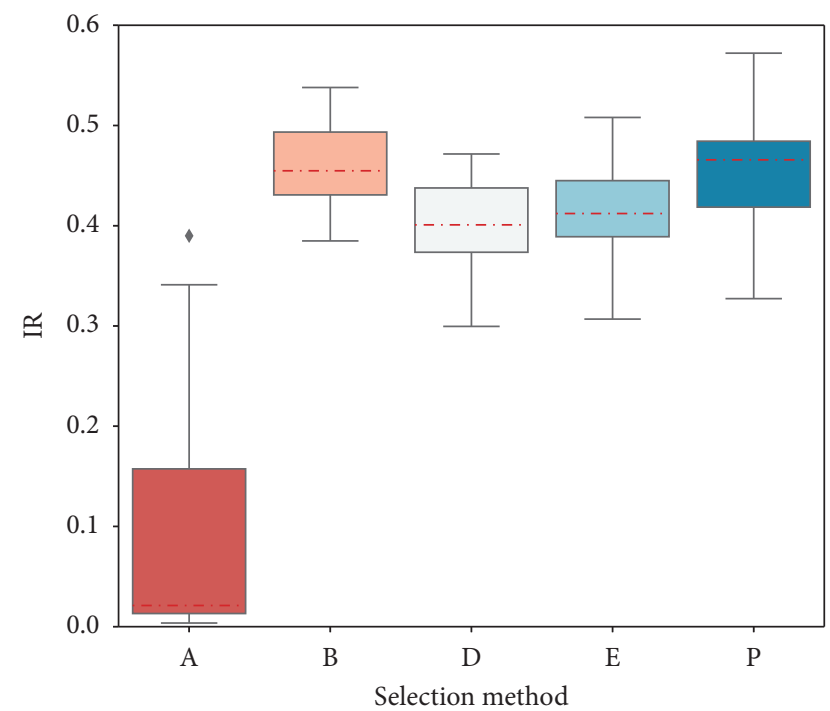

(b)

Figure 6: In L-Space for (a) STAR and (b) AVL, CFL, RGTR, and TICE networks. $p I=0.05, p R=0.0025$, and $T=65$ (boxplot diagram for A, $\mathrm{B}, \mathrm{D}, \mathrm{E}$, and $\mathrm{P}$ groups).

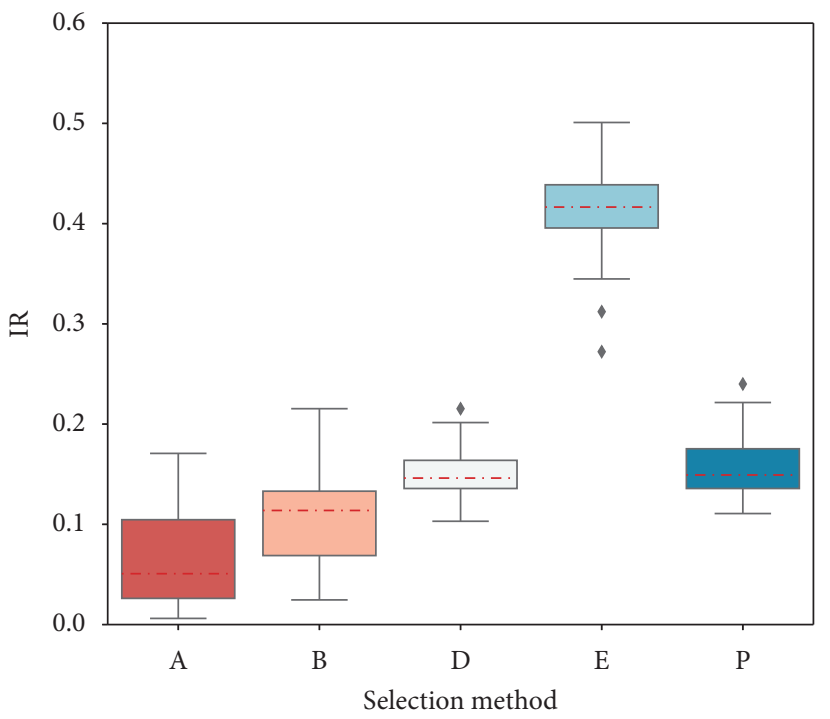

(a)

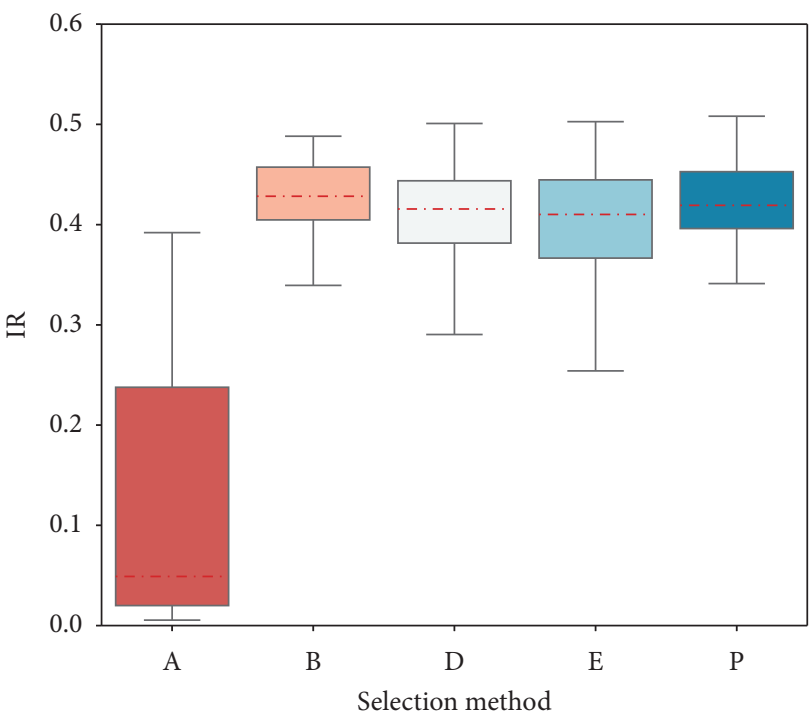

(b)

Figure 7: Continued. 


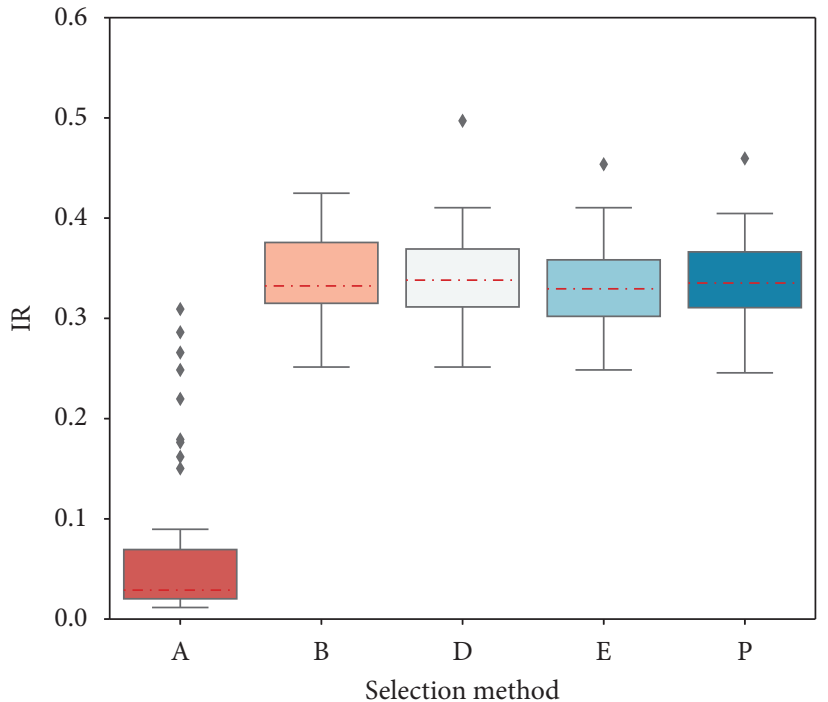

(c)

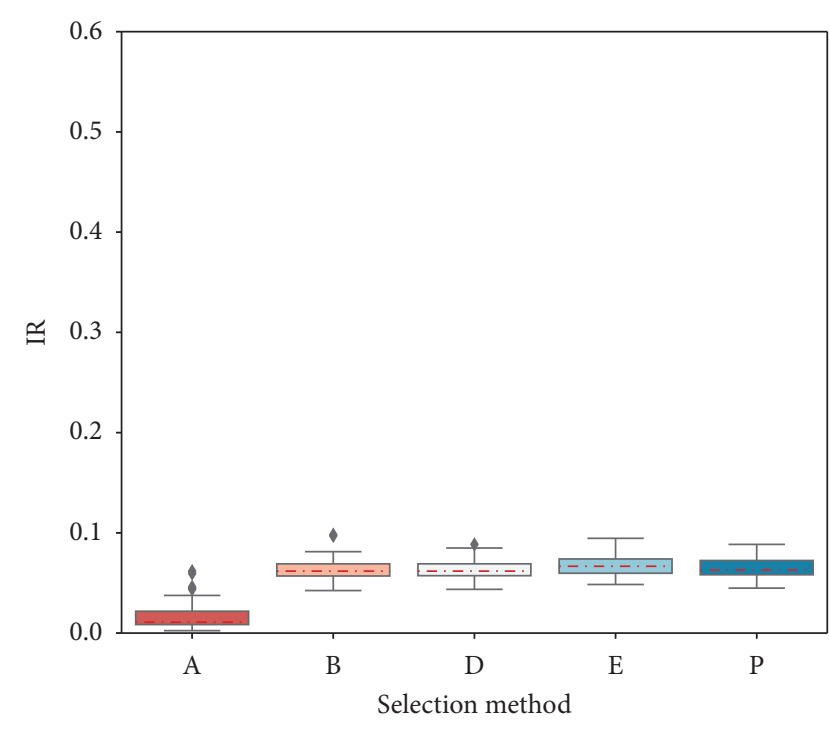

(d)

Figure 7: In L-Space for (a) TransAntofagasta, (b) Linja-Karjala Oy, (c) CIT, and (d) Thunder Bay Transit. $p I=0.05, p R=0.0025$, and $T=65$ (boxplot diagram for A, B, D, E, and P groups).

TABLE 7: In L-Space, for BC Transit network, $p I=0.05, p R=0.0025$, and $T=150, p$-value of the Wilcoxon rank sum test (resultant $p$-value per Bonferroni correction is indicated in brackets).

\begin{tabular}{lcccc}
\hline & $\mathrm{A}$ & $\mathrm{B}$ & $\mathrm{D}$ & $\mathrm{E}$ \\
\hline $\mathrm{B}$ & $<5.70 e-11(8.60 e-10)$ & $1.60 e-09(2.30 e-08)$ & & \\
$\mathrm{D}$ & $1.10 e-14(1.60 e-13)$ & $3.70 e-09(5.50 e-08)$ & $0.90000(1.00000)$ & \\
$\mathrm{E}$ & $2.40 e-14(3.50 e-13)$ & $1.30 e-08(2.00 e-07)$ & $0.27000(1.00000)$ & $0.34000(1.00000)$ \\
$\mathrm{P}$ & $2.20 e-14(3.30 e-13)$ & &
\end{tabular}

TABLE 8: In L-Space, for Kolumbus network, $p I=0.05, p R=0.0025$, and $T=150$, $p$-value of the Wilcoxon rank sum test (resultant $p$-value per Bonferroni correction is indicated in brackets).

\begin{tabular}{lcccc}
\hline & $\mathrm{A}$ & $\mathrm{B}$ & $\mathrm{D}$ & $\mathrm{E}$ \\
\hline $\mathrm{B}$ & $<1.40 e-13(2.00 e-12)$ & & & \\
$\mathrm{D}$ & $0.00473(0.071)$ & $<2.00 e-16(1.10 e-15)$ & & \\
$\mathrm{E}$ & $3.00 e-14(4.50 e-13)$ & $0.44174(1.000)$ & $<2.00 e-16(1.00 e-15)$ & $<2.00 e-16(1.00 e-15)$ \\
$\mathrm{P}$ & $0.00554(0.083)$ & $<2.00 e-16(1.10 e-15)$ & $0.99123(1.000)$ & $<$ \\
\hline
\end{tabular}

TABLE 9: In L-Space, for EMT network, $p I=0.05, p R=0.0025$, and $T=150$, $p$-value of in the Wilcoxon rank sum test (resultant $p$-value per Bonferroni correction is indicated in brackets).

\begin{tabular}{lcccc} 
& $\mathrm{A}$ & $\mathrm{B}$ & $\mathrm{D}$ & $\mathrm{E}$ \\
\hline $\mathrm{B}$ & $<2.00 e-16(<2.00 e-16)$ & & & \\
$\mathrm{D}$ & $<2.00 e-16(<2.00 e-16)$ & $0.00027(0.0041)$ & & \\
$\mathrm{E}$ & $<2.00 e-16(<2.00 e-16)$ & $1.40 e-06(2.00 e-05)$ & $0.30986(1.00000)$ & \\
$\mathrm{P}$ & $<2.00 e-16(<2.00 e-16)$ & $<2.00 e-16(2.00 e-16)$ & $<2.00 e-16(<2.00 e-16)$ & $<2.00 e-16(<2.00 e-16)$ \\
\hline
\end{tabular}

TABle 10: In L-Space, for Auckland network, $p I=0.05, p R=0.0025$, and $T=150$, $p$-value of the Wilcoxon rank sum test (resultant $p$-value per Bonferroni correction is indicated in brackets).

\begin{tabular}{lcccc}
\hline & $\mathrm{A}$ & $\mathrm{B}$ & $\mathrm{D}$ & $\mathrm{E}$ \\
\hline $\mathrm{B}$ & $<2.00 e-16(<2.00 e-16)$ & & & \\
$\mathrm{D}$ & $<2.00 e-16(<2.00 e-16)$ & $0.49000(1.00000)$ & & \\
$\mathrm{E}$ & $<2.00 e-16(<2.00 e-16)$ & $7.40 e-14(1.10 e-12)$ & $3.60 e-12(5.30 e-11)$ & \\
$\mathrm{P}$ & $<2.00 e-16(<2.00 e-16)$ & $0.54000(1.00000)$ & $0.96000(1.00000)$ & $1.00 e-12(1.50 e-11)$ \\
\hline
\end{tabular}


TABLE 11: In L-Space, for STAR network, $p I=0.05, p R=0.0025$, and $T=65, p$-value of the Wilcoxon rank sum test (resultant $p$-value per Bonferroni correction is indicated in brackets).

\begin{tabular}{lcccc}
\hline & $\mathrm{A}$ & $\mathrm{B}$ & $\mathrm{D}$ & $\mathrm{E}$ \\
\hline $\mathrm{B}$ & $1.20 e-10(1.20 e-09)$ & & & \\
$\mathrm{D}$ & $5.70 e-12(5.70 e-11)$ & $0.056(0.56)$ & $0.007(0.07000)$ & $0.01900(0.19000)$ \\
$\mathrm{E}$ & $2.90 e-14(2.90 e-13)$ & $0.752(1.00000)$ & $0.689(1.00000)$ & $0.193(1.00000)$ \\
$\mathrm{P}$ & $1.20 e-12(1.20 e-11)$ & & & \\
\hline
\end{tabular}

TABLE 12: In L-Space, for AVL, CFL, RGTR, and TICE networks, $p I=0.05, p R=0.0025$, and $T=65$, $p$-value of the Wilcoxon rank sum test (resultant $p$-value per Bonferroni correction is indicated in brackets).

\begin{tabular}{lcccc}
\hline & $\mathrm{A}$ & $\mathrm{B}$ & $\mathrm{D}$ & $\mathrm{E}$ \\
\hline $\mathrm{B}$ & $2.30 e-14(2.3 e-13)$ & & & \\
$\mathrm{D}$ & $2.00 e-16(4.8 e-16)$ & $1.70 e-07(1.7 e-06)$ & $0.19(1.00000)$ & \\
$\mathrm{E}$ & $<2.00 e-16(3.1 e-16)$ & $9.00 e-06(9.0 e-05)$ & $2.70 e-07(2.7 e-06)$ & $4.30 e-05(0.00043)$ \\
$\mathrm{P}$ & $<2.00 e-16(<2 e-16)$ & $0.19(1.00000)$ & & \\
\hline
\end{tabular}

TABle 13: In L-Space, for TransAntofagasta network, $p I=0.05, p R=0.0025$, and $T=65, p$-value of the Wilcoxon rank sum test (resultant $p$-value per Bonferroni correction is indicated in brackets).

\begin{tabular}{ccccc}
\hline & $\mathrm{A}$ & $\mathrm{B}$ & $\mathrm{D}$ & $\mathrm{E}$ \\
\hline $\mathrm{B}$ & $0.0013(0.008)$ & & & \\
$\mathrm{D}$ & $1.80 e-11(1.10 e-10)$ & $5.50 e-07(3.30 e-06)$ & & \\
$\mathrm{E}$ & $1.70 e-14(1.70 e-13)$ & $2.90 e-14(2.90 e-13)$ & $<2.00 e-16(<2.00 e-16)$ & $<2.00 e-16(<2.00 e-16)$ \\
$\mathrm{P}$ & $6.50 e-12(3.90 e-11)$ & $1.50 e-07(8.90 e-07)$ & $0.5348(1.00000)$ & $<$ \\
\hline
\end{tabular}

TABLe 14: In L-Space, for Linja-Karjala Oy network, $p I=0.05, p R=0.0025$, and $T=65, p$-value of the Wilcoxon rank sum test (resultant $p$-value per Bonferroni correction is indicated in brackets).

\begin{tabular}{lcccc}
\hline & $\mathrm{A}$ & $\mathrm{B}$ & $\mathrm{D}$ & $\mathrm{E}$ \\
\hline $\mathrm{B}$ & $1.10 e-13(1.10 e-12)$ & $0.11600(1.00000)$ & & \\
$\mathrm{D}$ & $2.40 e-16(2.40 e-15)$ & $0.11000(1.00000)$ & $0.59500(1.00000)$ & $0.09800(0.98000)$ \\
$\mathrm{E}$ & $1.50 e-15(1.50 e-14)$ & $0.65500(1.00000)$ & $0.16400(1.00000)$ & 0 \\
$\mathrm{P}$ & $<2.00 e-16(3.20 e-16)$ & &
\end{tabular}

TABLE 15: In L-Space, for CIT network, $p I=0.05, p R=0.0025$, and $T=65$, $p$-value of the Wilcoxon rank sum test (resultant $p$-value per Bonferroni correction is indicated in brackets).

\begin{tabular}{lcccc}
\hline & $\mathrm{A}$ & $\mathrm{B}$ & $\mathrm{D}$ & $\mathrm{E}$ \\
\hline $\mathrm{B}$ & $1.30 e-13(1.30 e-12)$ & & & \\
$\mathrm{D}$ & $<2.00 e-16(7.50 e-16)$ & $1.00000(1.00000)$ & $0.20000(1.00000)$ & \\
$\mathrm{E}$ & $<2.00 e-16(1.40 e-15)$ & $0.29000(1.00000)$ & $0.56(1.00000)$ & $0.41(1.00000)$ \\
$\mathrm{P}$ & $<2.00 e-16(7.30 e-16)$ & $0.63(1.00000)$ & \\
\hline
\end{tabular}

TABLE 16: In L-Space, for Thunder Bay Transit network, $p I=0.05, p R=0.0025$, and $T=65, p$-value of the Wilcoxon rank sum test (resultant $p$-value per Bonferroni correction is indicated in brackets).

\begin{tabular}{lcccc}
\hline & $\mathrm{A}$ & $\mathrm{B}$ & $\mathrm{D}$ & $\mathrm{E}$ \\
\hline $\mathrm{B}$ & $3.00 e-13(3.00 e-12)$ & & & \\
$\mathrm{D}$ & $<2.00 e-16(1.50 e-15)$ & $0.47900(1.00000)$ & $0.08300(0.83000)$ & \\
$\mathrm{E}$ & $<2.00 e-16(5.60 e-16)$ & $0.02700(0.27000)$ & $0.44800(1.00000)$ & $0.27900(1.00000)$ \\
$\mathrm{P}$ & $<2.00 e-16(9.30 e-16)$ & $0.16900(1.00000)$ & \\
\hline
\end{tabular}


TABLE 17: For $p I=0.05$ and $p R=0.0025$, average propagation time in which IR reached $60 \%$ in each group $(\mathrm{A}, \mathrm{B}, \mathrm{D}, \mathrm{E}, \mathrm{P})$ and globally.

\begin{tabular}{|c|c|c|c|c|c|c|}
\hline & $\mathrm{A}$ & $\mathrm{B}$ & $\mathrm{D}$ & $E$ & $\mathrm{P}$ & All \\
\hline Auckland & 542.13 & 386.85 & 389.85 & 408.83 & 389.37 & 415.24 \\
\hline EMT & 261.31 & 171.75 & 162.83 & 162.06 & 195.33 & 202.99 \\
\hline BC Transit & $>700$ & $>700$ & $>700$ & $>700$ & $>700$ & $>700$ \\
\hline Kolumbus & 552.99 & 458.23 & $>700$ & 489.01 & $>700$ & $>700$ \\
\hline STAR & 116.75 & 93.51 & 99.74 & 95.09 & 97.88 & 99.67 \\
\hline AVL, CFL, RGTR, and TICE & 121.37 & 84.42 & 90.43 & 89.58 & 83.73 & 91.51 \\
\hline TransAntofagasta & $>150$ & 145 & $>150$ & 101.16 & $>150$ & 105.57 \\
\hline Linja-Karjala Oy & 127.22 & 98.64 & 102.54 & 102.12 & 97.50 & 103.78 \\
\hline CIT & 140.00 & 115.65 & 117.98 & 119.56 & 120.18 & 120.01 \\
\hline Thunder Bay Transit & $>150$ & $>150$ & $>150$ & $>150$ & $>150$ & $>150$ \\
\hline
\end{tabular}

TABLE 18: For $p I=0.05$ and $p R=0.0025$, median propagation time in which IR reached $60 \%$ in each group $(A, B, D, E, P)$ and globally.

\begin{tabular}{|c|c|c|c|c|c|c|}
\hline & A & $\mathrm{B}$ & $\mathrm{D}$ & $\mathrm{E}$ & $\mathrm{P}$ & All \\
\hline Auckland & 545 & 385 & 390 & 409 & 388 & 399 \\
\hline EMT & 249.50 & 169 & 164 & 161 & 193 & 181 \\
\hline BC Transit & $>700$ & $>700$ & $>700$ & $>700$ & $>700$ & $>700$ \\
\hline Kolumbus & 547 & 455 & $>700$ & 486 & $>700$ & 486.50 \\
\hline STAR & 112.50 & 94 & 98 & 95 & 99 & 99.67 \\
\hline AVL, CFL, RGTR, and TICE & 116.00 & 84 & 89 & 90 & 81.50 & 88 \\
\hline TransAntofagasta & $>150$ & 149 & $>150$ & 100 & $>150$ & 101 \\
\hline Linja-Karjala Oy & 128 & 97 & 103 & 103 & 97 & 102 \\
\hline CIT & 138 & 113 & 117 & 119 & 120 & 119 \\
\hline Thunder Bay Transit & $>150$ & $>150$ & $>150$ & $>150$ & $>150$ & $>150$ \\
\hline
\end{tabular}

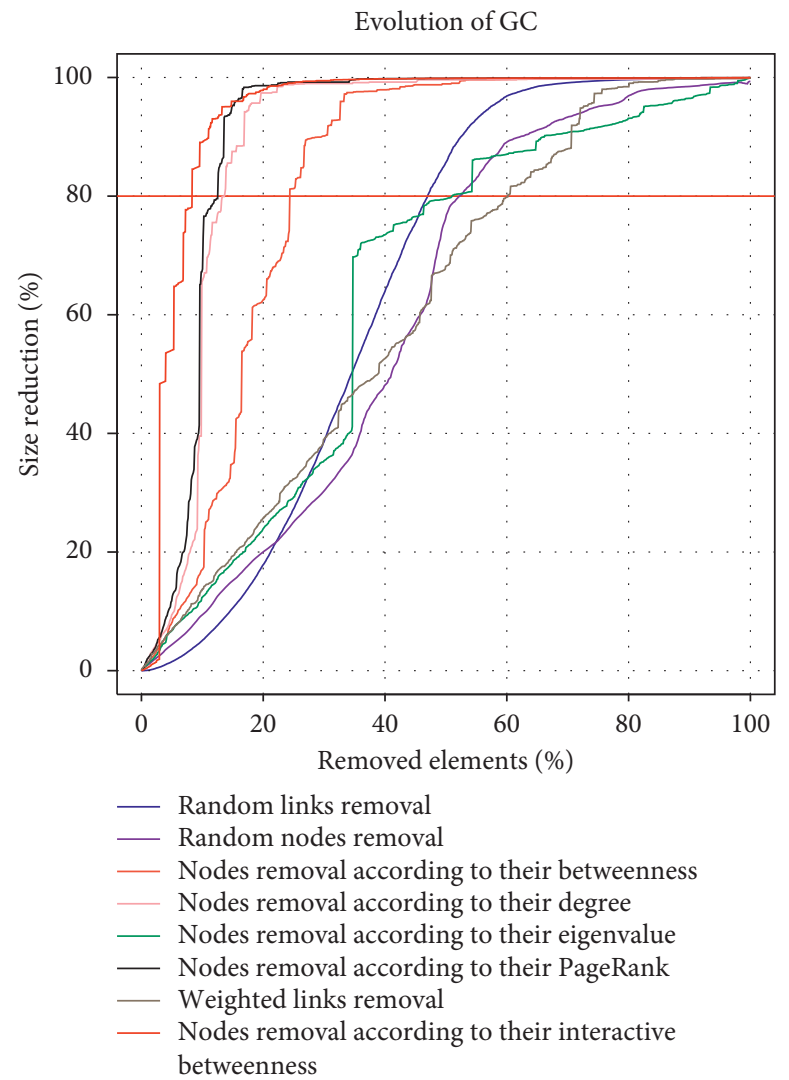

(a)

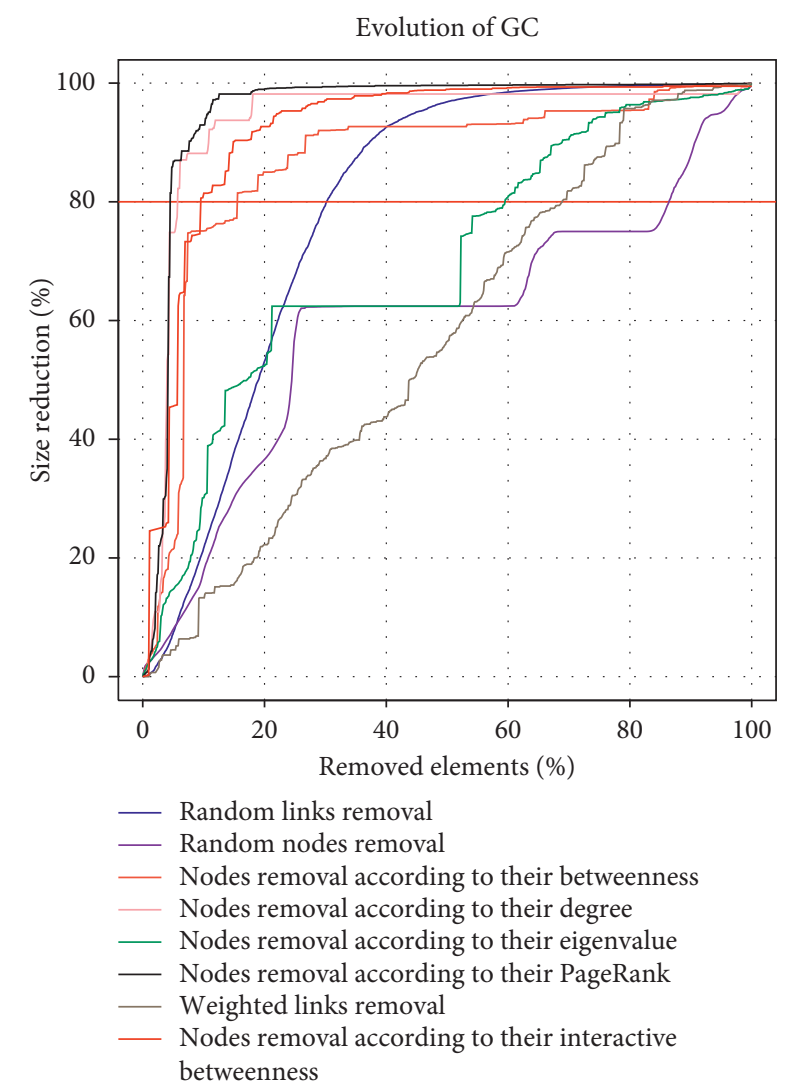

(b)

Figure 8: Continued. 
Evolution of GC

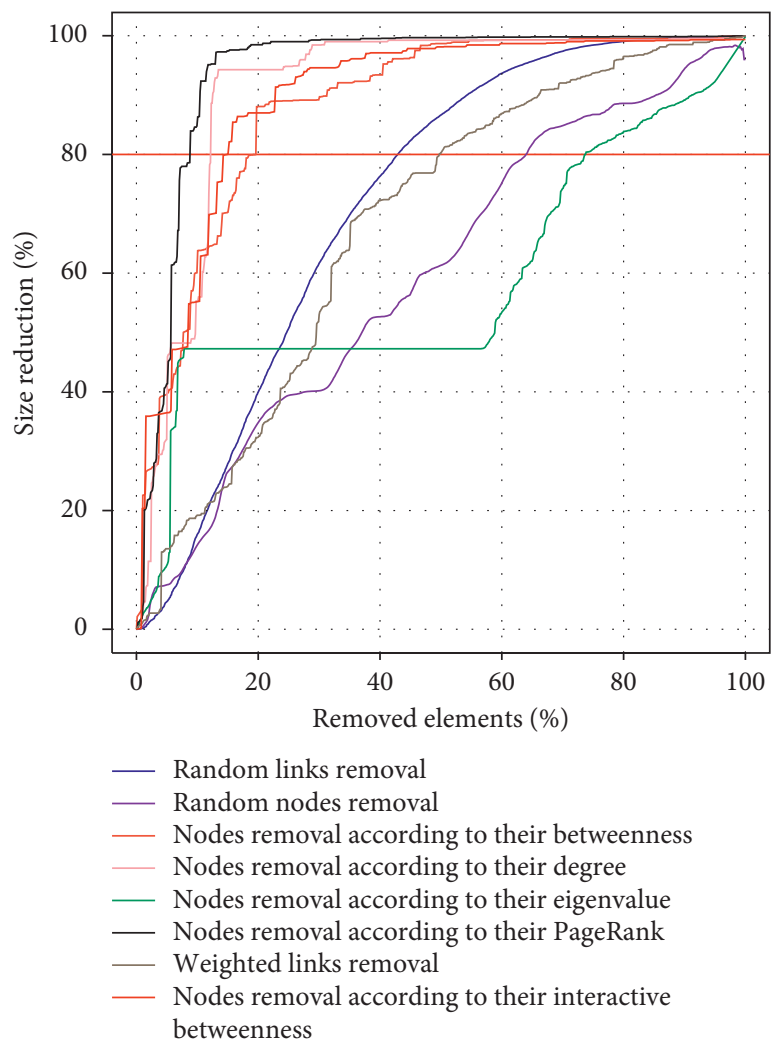

(c)
Evolution of GC

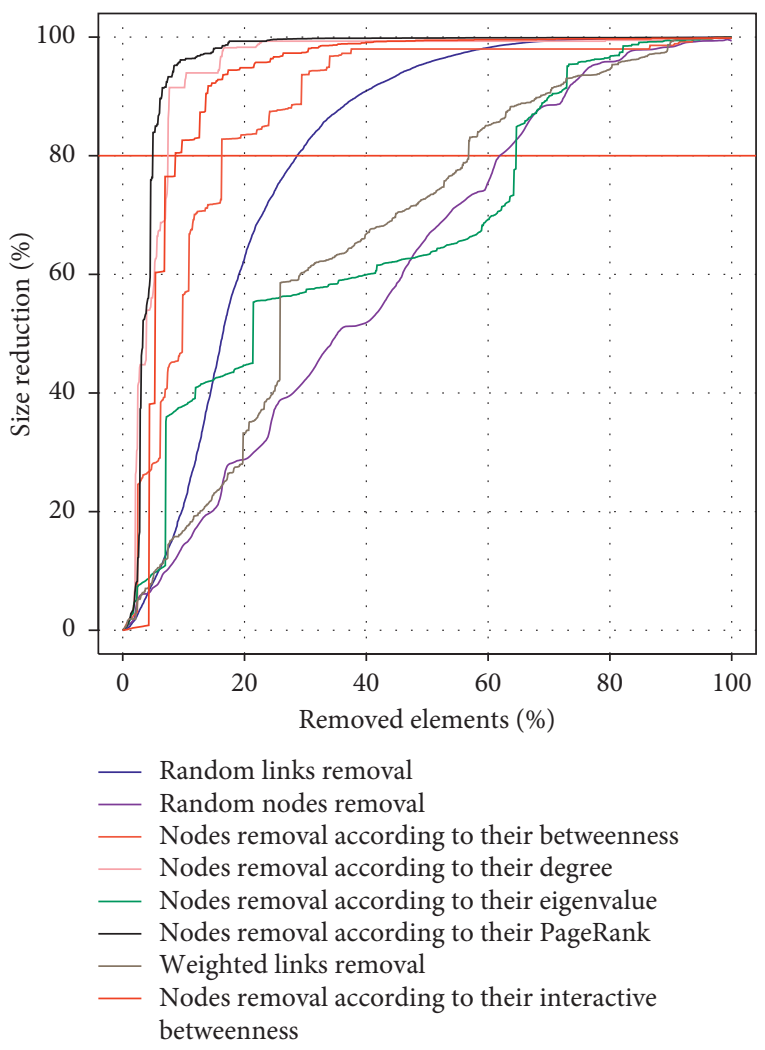

(d)

Figure 8: Evolution of the GC in EMT (a), BC Transit (b), Kolumbus (c), and Auckland (d) networks.
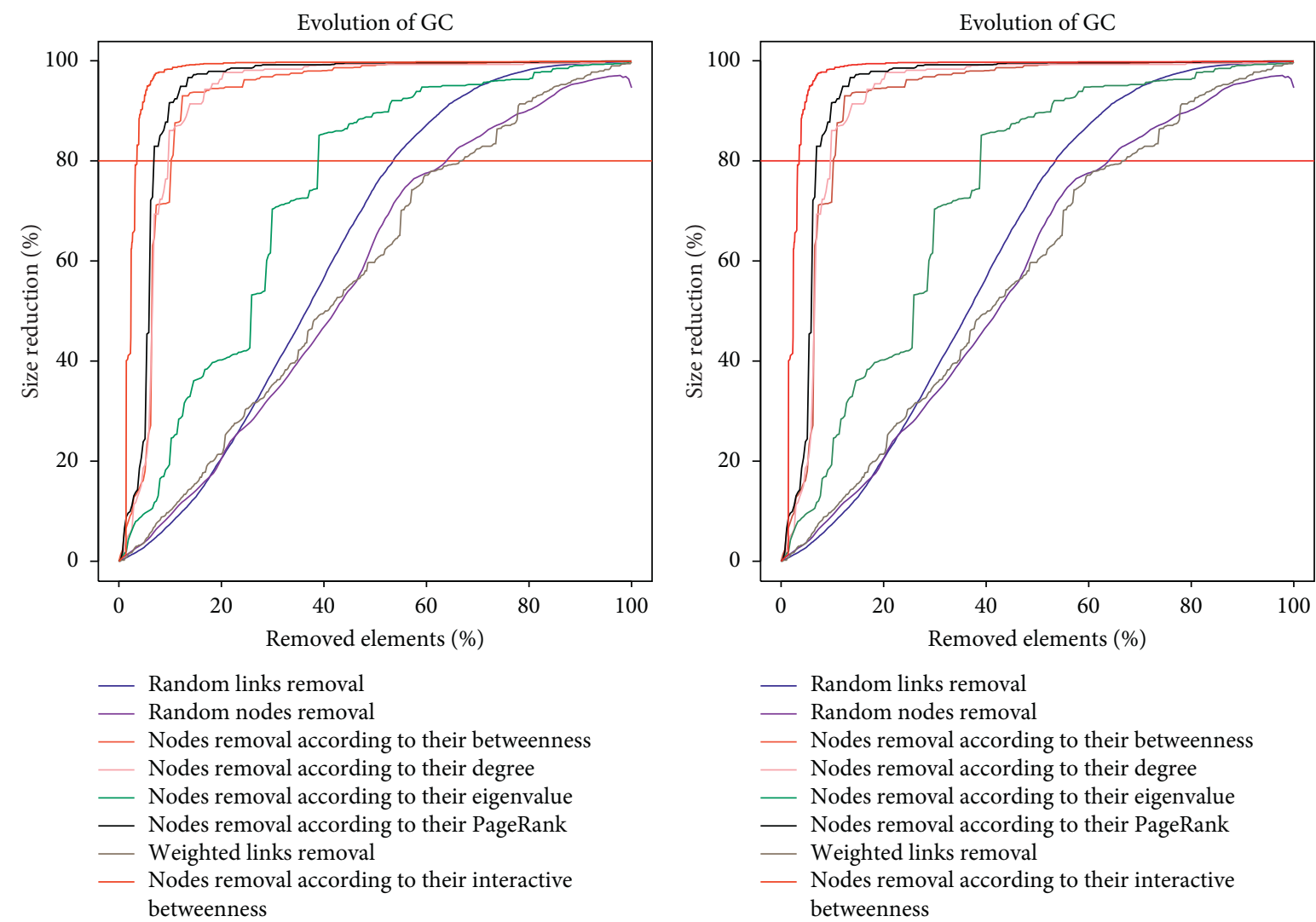

(a) 

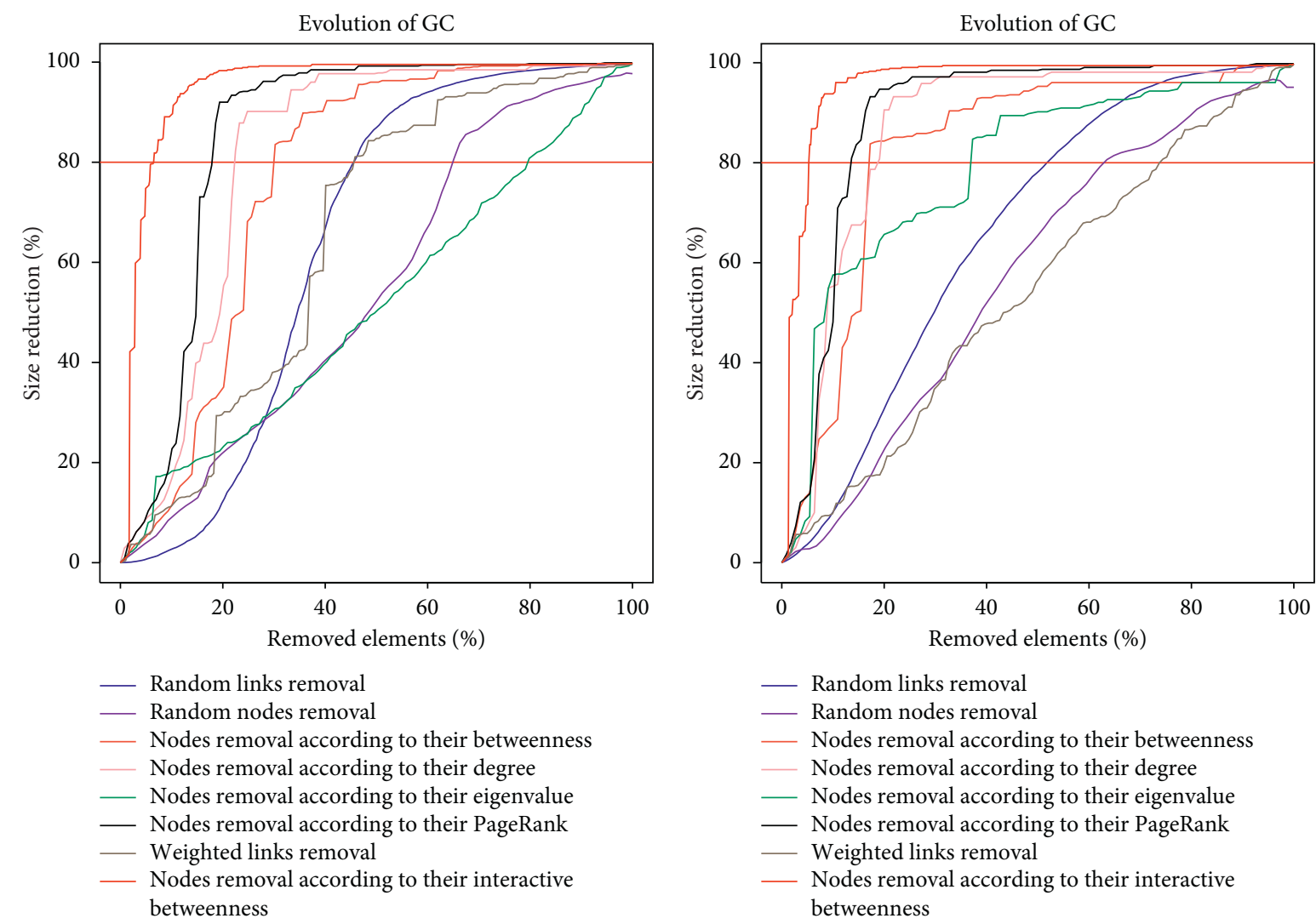

(c)

(d)

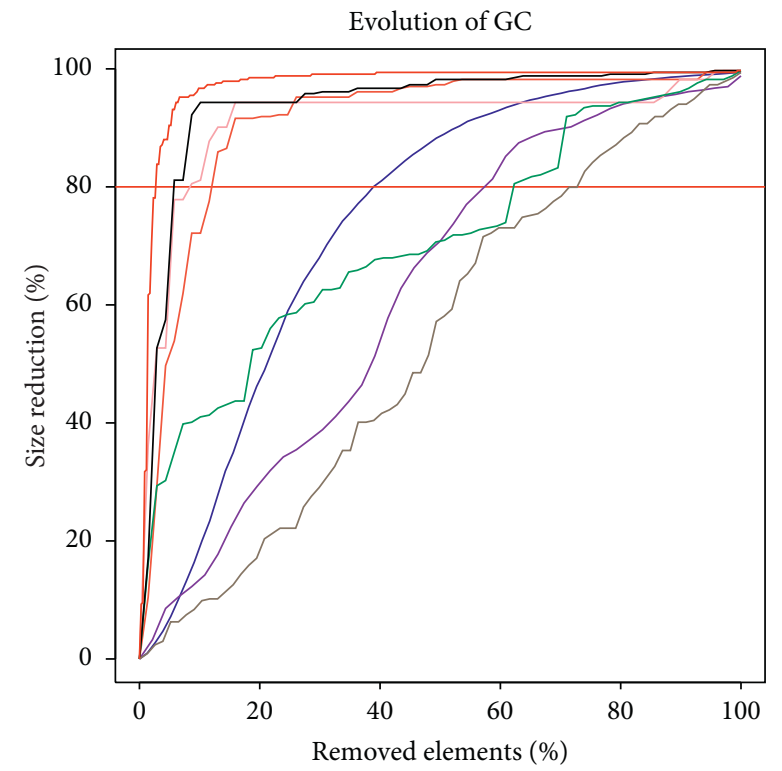

Evolution of GC

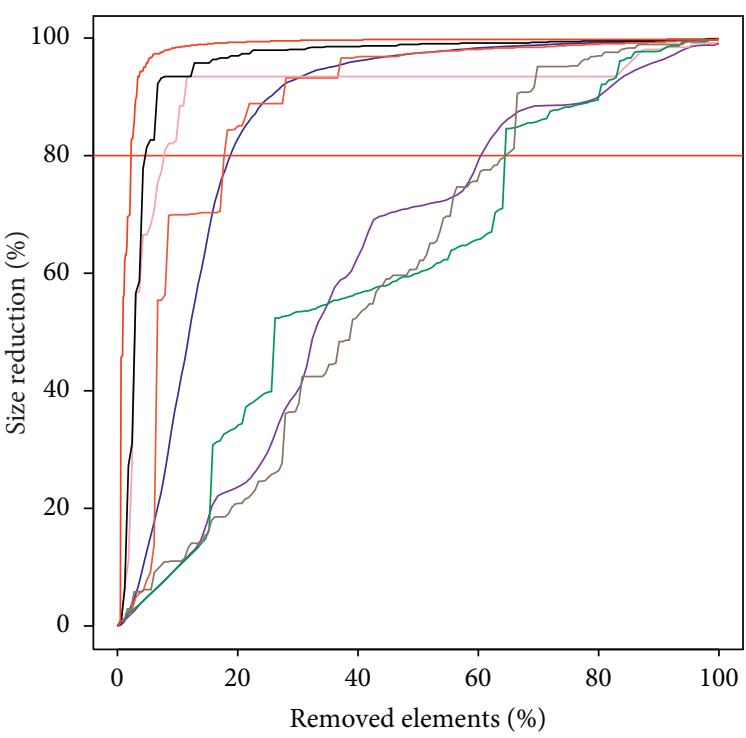

Random links removal

Random nodes removal

- Nodes removal according to their betweenness

- Nodes removal according to their degree

- Nodes removal according to their eigenvalue

- Nodes removal according to their PageRank

Weighted links removal

- Nodes removal according to their interactive betweenness

(e)

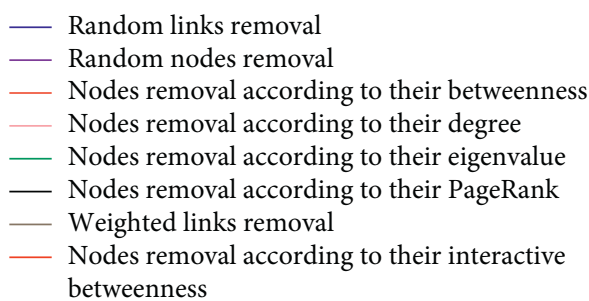

(f)

Figure 9: Evolution of the GC in AVL, CFL, RGTR, and TICE (a), STAR (b), TransAntofagasta (c), Linja-Karjala Oy (d), CIT ChamblyRichelieu-Carignan network (e), and Thunder Bay Transit (f). 
TABLE 19: Percentage of removed nodes with which the $80 \%$ of reduction in the GC is achieved.

\begin{tabular}{|c|c|c|c|c|c|}
\hline Network & Method & $\begin{array}{c}\% \text { of removed } \\
\text { elements }\end{array}$ & Network & Method & $\begin{array}{c}\% \text { of removed } \\
\text { elements }\end{array}$ \\
\hline \multirow[t]{8}{*}{ Auckland } & Pagerank centrality & 4.98 & EMT & $\begin{array}{l}\text { Interactive betweenness } \\
\text { centrality }\end{array}$ & 8.35 \\
\hline & Degree & 7.47 & & Pagerank centrality & 12.62 \\
\hline & $\begin{array}{c}\text { Interactive betweenness } \\
\text { centrality }\end{array}$ & 8.67 & & Degree & 13.70 \\
\hline & Betweenness centrality & 16.28 & & Betweenness centrality & 24.38 \\
\hline & Random links & 28.79 & & Random links & 47.02 \\
\hline & Weighted links & 56.85 & & Eigenvector centrality & 51.02 \\
\hline & Random nodes & 61.99 & & Random nodes & 52.42 \\
\hline & Eigenvector centrality & 64.66 & & Weighted links & 60.22 \\
\hline \multirow[t]{8}{*}{ BC Transit } & Pagerank centrality & 4.52 & Kolumbus & Pagerank centrality & 8.89 \\
\hline & Degree & 5.79 & & Degree & 12.29 \\
\hline & $\begin{array}{c}\text { Interactive betweenness } \\
\text { centrality }\end{array}$ & 9.55 & & $\begin{array}{l}\text { Interactive betweenness } \\
\text { centrality }\end{array}$ & 15.04 \\
\hline & Betweenness centrality & 15.58 & & Betweenness centrality & 19.74 \\
\hline & Random links & 30.19 & & Random links & 43 \\
\hline & Eigenvector centrality & 59.55 & & Weighted links & 49.75 \\
\hline & Weighted links & 68.94 & & Random nodes & 64.17 \\
\hline & Random nodes & 86.50 & & Eigenvector centrality & 73.86 \\
\hline \multirow[t]{8}{*}{ STAR } & $\begin{array}{c}\text { Interactive betweenness } \\
\text { centrality }\end{array}$ & 4.53 & $\begin{array}{l}\text { AVL, CFL, RGTR, } \\
\text { TICE }\end{array}$ & $\begin{array}{l}\text { Interactive betweenness } \\
\text { centrality }\end{array}$ & 3.57 \\
\hline & Pagerank & 14.18 & & pagerank & 6.93 \\
\hline & Degree & 15.60 & & Degree & 9.85 \\
\hline & Betweenness centrality & 17.73 & & Betweenness centrality & 10.22 \\
\hline & Eigenvector centrality & 45.74 & & Random links & 53.51 \\
\hline & Random links & 55.53 & & Eigenvector centrality & 39.05 \\
\hline & Weighted links & 64.07 & & Random nodes & 64.25 \\
\hline & Random nodes & 78.49 & & Weighted links & 66.49 \\
\hline \multirow[t]{8}{*}{ TransAntofagasta } & $\begin{array}{c}\text { Interactive betweenness } \\
\text { centrality }\end{array}$ & 6.63 & Linja-Karjala Oy & $\begin{array}{l}\text { Interactive betweenness } \\
\text { centrality }\end{array}$ & 5.27 \\
\hline & Pagerank & 17.83 & & Pagerank & 13.64 \\
\hline & Degree & 22.48 & & Betweenness centrality & 17.27 \\
\hline & Betweenness centrality & 30.23 & & Degree & 19.09 \\
\hline & Random links & 45.31 & & Eigenvector centrality & 37.27 \\
\hline & Weighted links & 45.83 & & Random links & 52.48 \\
\hline & Random nodes & 65.12 & & Random nodes & 63.51 \\
\hline & Eigenvector centrality & 79.84 & & Weighted links & 73.76 \\
\hline \multirow[t]{8}{*}{ CIT } & $\begin{array}{l}\text { Interactive betweenness } \\
\text { centrality }\end{array}$ & 2.90 & $\begin{array}{c}\text { Thunder Bay } \\
\text { Transit }\end{array}$ & $\begin{array}{l}\text { Interactive betweenness } \\
\text { centrality }\end{array}$ & 2.31 \\
\hline & Pagerank & 5.80 & & Pagerank & 4.88 \\
\hline & Degree & 8.70 & & Degree & 7.93 \\
\hline & Betweenness centrality & 13.04 & & Betweenness centrality & 18.29 \\
\hline & Random links & 38.96 & & Random links & 18.99 \\
\hline & Random nodes & 58.70 & & Random nodes & 61.11 \\
\hline & Eigenvector centrality & 62.32 & & Eigenvector centrality & 64.63 \\
\hline & Weighted links & 74.03 & & Weighted links & 96.97 \\
\hline
\end{tabular}

\section{Conclusions}

An efficient PTN must be robust to provide a good quality service, even when it faces faults or disaster. This study analyses several PTNs, applying a methodology based on the study of structural properties, simulations of faults propagation, and the examination of the evolution of the GC. The analysis aims to detect the most vulnerable nodes and links when some faults occurred in them. The influence of the topological properties of the networks over their robustness has also been checked. This could allow preventative action in certain nodes and links against traffic congestion and unattainable stops, occurring as a result of construction work and catastrophes.

Problems that happened at random nodes and over the strongest links spread slower than other types of failure. Those nodes whose failures spread more quickly depended on the network, but they were those with the highest betweenness centrality, degree, or PageRank. All networks show high modularity $>0.8$ and a Molloy-Reed parameter $>2$. Additionally, all nodes were characterized by low $k$-core.

The AVL, CFL, RGTR, and TICE networks were that presented the highest-level propagation in a time $T$, for all 
analysed pairs $(p I, p R)$, followed in descending order by STAR, Linja-Karjala Oy, and TransAntofagasta. In the majority of the networks, $80 \%$ reduction in the size of GC with the minor percentage of removed nodes is reached for those nodes with the highest betweenness calculated interactively. In a small number of networks, this was achieved with the removal of nodes with the highest PageRank. The networks that showed the highest amount of sensitivity were in sequential order of Thunder Bay Transit; CIT; and AVL, CFL, RGTR, and TICE, with $2.31 \%, 2.90 \%$, and $3.57 \%$ of deleted nodes, respectively. In all networks, random failures took the longest time to spread across all networks, which proves, in conjunction with some structural properties ( $k$, PageRank, and eigenvector in L-Space), that the networks are less vulnerable to such failures.

This research can be continued by analysing passenger flow in the PTN as a dynamically changing characteristic. Some models that take into account the individual passengers' behaviour, with respect to the choice of routes and connections during a trip, can be implemented in order to dynamically establish the possible consequences of the congestion. It is also possible to study how the passengers modified their travel behaviour when considering their previous experience. Additionally, the PTN vulnerability can be examined in other graph representations, as well as considering different link weights, such as trip time or financial costs. In addition to the above, in a catastrophic situation, the postdisaster circumstances can be examined, completing the analysis of the evolution of GC with a study of the access to critical facilities (i.e., emergency services).

\section{Data Availability}

Information of stops and routes of Auckland, EMT, BC Transit, Kolumbus, STAR, AVL, CFL, RGTR, TICE, Thunder Bay Transit, TransAntofagasta, Linja-Karjala Oy, and CIT networks were retrieved from the operating companies public web sites, the Deconet Public Transport Network Data and GTFS Data Exchange repositories

\section{Conflicts of Interest}

The author declares that there are no conflicts of interest regarding the publication of this paper.

\section{Acknowledgments}

The author would like to thank Dr. Mari Luz Congosto Martínez for her guidance on data visualization. This research was partially funded by Telefónica Chair at Universidad Francisco de Vitoria.

\section{References}

[1] C. Wan, Z. Yang, D. Zhang, X. Yan, and S. Fan, "Resilience in transportation systems: a systematic review and future directions," Transport Reviews, vol. 38, no. 4, 2018.

[2] C. Wan, Z. Yang, Z. Xu, W. Zhang, and T. Zheng, "Space P-based empirical research on public transport complex networks in 330 cities of China," Reliability Engineering and System Safety, vol. 13, no. 1, 2013.
[3] F. C. Von Feber, T. Holovatch, Y. Holovatch, and V. Palchykov, "Public transport networks: empirical analysis and modelling," European Physical Journal B, vol. 68, 2009.

[4] B. Berche, C. von Ferber, T. Holovatch, and Y. Holovatch, "Resilience of public transport networks against attacks," Physics of Condensed Matter, vol. 71, 2009.

[5] O. Lordan, J. M. Sallan, P. Simo, and D. González-Prieto, "Robustness of the air transport network," Transportation Research Part E: Logistics and Transportation Review, vol. 68, 2014.

[6] S. Wandelt, X. Sun, D. Feng, M. Zanin, and S. Havlin, "A comparative analysis of approaches to network-dismantling," Scientific Reports, vol. 8, 2018.

[7] S. Wandelt, X. Shi, and X. Sun, "Approximation of interactive betweenness centrality in large complex networks," Complexity, vol. 2020, Article ID 4046027, 2020.

[8] M. D. Yap, N. Van Oort, R. Nes, and B. Arem, "Identification and quantification of link vulnerability in multi-level public transport networks: a passenger perspective"' Transportation, vol. 45, 2018.

[9] E. Jenelius and O. Cats, "The value of new public transport links for network robustness and redundancy," Quantitative Approaches to Resilience in Transport Networks, vol. 11, no. 9, 2015.

[10] X. Albacete, D. Olaru, V. Paül, and S. Biermann, "Measuring the accessibility of public transport: a critical comparison between methods in Helsinki," Applied Spatial Analysis and Policy, vol. 10, no. 2, 2017.

[11] F. Corman F, A. D’Ariano, and I. A. Hansen, "Evaluating disturbance robustness of railway schedules," Journal of Intelligent Transportation Systems, vol. 18, no. 1, 2014.

[12] S. Wandelt, X. Shi, and X. Sun, "Estimation and improvement of transportation network robustness by exploiting communities," Reliability Engineering \& System Safety, vol. 206, 2021.

[13] X. Sun, S. Wandelt, and A. Zhang, "Resilience of cities towards airport disruptions at global scale," Research in Transportation Business \& Management", vol. 34, 2020.

[14] A. Chen, C. Yang, S. Kongsomsaksakul, and M. Lee, "Network-based accessibility measures for vulnerability analysis of degradable transportation networks," Networks and Spatial Economics, vol. 7, 2007.

[15] F. Liao and B. Van Wee, "Accessibility measures for robustness of the transport system," Transportation, vol. 44, no. 5, 2017.

[16] Z. He, J. N. Guo, and J. X. Xu, "Cascade failure model in multimodal transport network risk propagation," Mathematical Problems in Engineering, vol. 12, 2019.

[17] E. Baspinar and B. Koyuncu, "A data-driven air transportation delay propagation model using epidemic process models"' International Journal of Aerospace Engineering, vol. 2016, 2016.

[18] M. Akdere, C. C. Bilgin, O. Gerdaneri, I. Korpeoglu, Ö. Ulusoy, and U. Çetintemel, "A comparison of epidemic algorithms in wireless sensor networks"” Journal Computer Communications, vol. 3, no. 2, 2006.

[19] M. Nekovee, "Epidemic algorithms for reliable and eficient information dissemination in vehicular ad hoc networks," IET Intelligent Transport Systems, vol. 3, no. 2, 2009.

[20] R Project, $R$ Project for Statistical Computing, https://www.rproject.org/, 2020.

[21] Python, Python Lenguaje de programación, https://www. python.org/, 2020. 
[22] V. Latora and M. Marchiori, "Efficient behavior of smallworld networks," Physical Review Letters, vol. 87, no. 19, 2001.

[23] P. Angeloudis and D. Fisk, "Large subway systems as complex networks," Physica A: Statistical Mechanics and its Applications, vol. 367, 2006.

[24] B. Hu, Y. Pei, J. Tang, and W. Gao, "Common network characteristics of four bus transport networks in Northeast China based on a perfect space P," International Journal of Modern Physics B, vol. 32, no. 21, 2018.

[25] K. Ma, Z. W. Wang, J. W. Jiang, G. X. Zhu, and W. Li, "Power law and small world properties in a comparison of traffic city networks," Chinese Science Bulletin, vol. 56, no. 34, 2011.

[26] M. E. J. Newman, "The structure and function of complex networks," SIAM Review, vol. 45, no. 2, 2003.

[27] E. Estrada, D. Higham, and N. Hatano, "Communicability betweenness in complex networks," Physica A: Statistical Mechanics and its Applications, vol. 388, no. 5, 2009.

[28] M. Dehmer, Structural Analysis of Complex Networks", Birkhäuser, Vienna, Austria, 2011.

[29] C. Durón, "Heatmap centrality: a new measure to identify super-spreader nodes in scale-free networks," PLoS One, vol. 15 , no. $7,2020$.

[30] N. Perra and F. Santo, "Spectral centrality measures in complex networks," Physical Review E, Statistical, Nonlinear, and Soft Matter Physics, vol. 78, 2008.

[31] E. Becker, B. Robisson, C. Chapple, A. Guenoche, and C. Brun, "Multifunctional proteins revealed by overlapping clustering in protein interaction network," Bioinformatics, vol. 28 , no. $1,2012$.

[32] M. Newman, "Assortative mixing in networks," Physical Review Letters, vol. 89, no. 20, 2002.

[33] A. L. Barabasi, Network Science Network Robustness, https:// barabasi.com/f/619.pdf, 2014.

[34] D. J. Sheskin, Handbook of Parametric and Nonparametric Statistical Procedures, CRC Press, Wallingford, CT, USA., 2011.

[35] R. Khan and F. Ahmad, "Power comparison of various normality tests," Pakistan Journal of Statistics and Operation Research, vol. 11, no. 3, 2017.

[36] A. Halunga, C. D. Orme, and T. Yamagata, Handbook of Parametric and Nonparametric Statistical Procedures, School of Social Sciences, University of Manchester, Manchester, UK, 2011.

[37] M. Neuhauser, Nonparametric Statistical Tests: A Computational Approach, Chapman and Hall/CRC, New York, NY, USA, 2017.

[38] V. Romano, M. Shen, J. Pansanel et al., "Social transmission in networks: global efficiency peaks with intermediate levels of modularity," Behav Ecol Sociobiol, vol. 72, no. 154, 2018.

[39] I. McCulloh, "Network topology effects on correlation between centrality measures," Connections, vol. 30, no. 1, pp. 21-28, 2010.

[40] S. Ferreira, C. Rodrigues, and F. Fagundes, "Structure and robustness of Sao Paulo public transport network," Physics and Society, vol. 4, no. 3, 2018.

[41] J. Borge-Holthoefer and Y. Moreno, "Absence of influential spreaders in rumour dynamics," Physical Review E, Statistical, Nonlinear, and Soft Matter Physics, vol. 85, no. 2, 2012. 